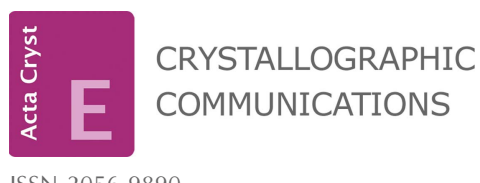

ISSN 2056-9890

Received 11 April 2017

Accepted 27 April 2017

Edited by T. J. Prior, University of Hull, England

Keywords: crystal structure; carboxamide; pyrazine; pyridine; silver(I); Ag-Ag bond; three-dimensional coordination polymer; hydrogen bonding.

CCDC references: $1546645 ; 1546644$

Supporting information: this article has supporting information at journals.iucr.org/e

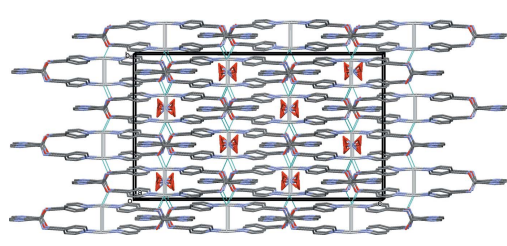

OPEN $\odot$ ACCESS

\section{Crystal structure of a pyrazine-2,3-dicarboxamide ligand and of its silver(I) nitrate complex, a three- dimensional coordination polymer}

\author{
Dilovan S. Cati ${ }^{\mathrm{a}}$ and Helen Stoeckli-Evans ${ }^{\mathrm{b} *}$
}

aDebiopharm International S.A., Chemin Messidor 5-7, CP 5911, CH-1002 Lausanne, Switzerland, and ${ }^{\mathbf{b}}$ Institute of
Physics, University of Neuchâtel, rue Emile-Argand 11, CH-2000 Neuchâtel, Switzerland. *Correspondence e-mail:
helen.stoeckli-evans@unine.ch

The title ligand, $\mathrm{C}_{18} \mathrm{H}_{16} \mathrm{~N}_{6} \mathrm{O}_{2} \cdot 2 \mathrm{H}_{2} \mathrm{O}$ (L1) $\left[N^{2}, N^{3}\right.$-bis(pyridin-4-ylmethyl)pyrazine-2,3-dicarboxamide], crystallized as a dihydrate. The molecule is Ushaped with the carboxamide groups being cis to one another, making a dihedral angle of $81.6(5)^{\circ}$. The terminal pyridine rings are inclined to one another by $58.5(4)^{\circ}$. There is an intramolecular $\mathrm{N}-\mathrm{H} \cdots \mathrm{N}_{\text {pyrazine }}$ hydrogen bond present, forming an $S(5)$ ring motif. In the crystal, adjacent molecules are linked by $\mathrm{N}-$ $\mathrm{H} \cdots \mathrm{O}_{\text {carboxamide }}$ hydrogen bonds, forming a chain along [001]. A chain of hydrogen-bonded water molecules is linked to the chain of $(\mathbf{L 1})$ molecules by $\mathrm{O}-\mathrm{H} \cdots \mathrm{N}$ hydrogen bonds, forming columns propagating along the $c$ axis. The columns are linked by $\mathrm{C}-\mathrm{H} \cdots \mathrm{O}$ and $\mathrm{C}-\mathrm{H} \cdots \mathrm{N}$ hydrogen bonds, forming a three-dimensional supramolecular structure. The reaction of ligand (L1) with silver(I) nitrate led to the formation of a new three-dimensional coordination polymer, $\quad\left\{\left[\mathrm{Ag}\left(\mathrm{C}_{18} \mathrm{H}_{16} \mathrm{~N}_{6} \mathrm{O}_{2}\right)\right] \mathrm{NO}_{3}\right\}_{n}, \quad$ poly $\left[\left[\left[\mu_{4}-N^{2}, N^{3}\right.\right.\right.$-bis(pyridin-4-ylmethyl)pyrazine-2,3-dicarboxamide]silver(I)] nitrate] (I). The asymmetric unit is composed of half of one silver ion, located on a twofold rotation axis, half a ligand molecule and half a positionally disordered nitrate anion located about a twofold rotation axis. The full molecule of the ligand is generated by twofold rotational symmetry, with this twofold axis bisecting the $\mathrm{C}_{\mathrm{ar}}-\mathrm{C}_{\mathrm{ar}}$ bonds of the pyrazine ring and the $\mathrm{Ag}-\mathrm{Ag}$ bond. The carboxamide groups are now trans to one another, making a dihedral angle of $65.8(4)^{\circ}$. The two terminal pyridine rings are inclined to one another by $6.6(3)^{\circ}$. Two ligands wrap around an $\mathrm{Ag}-$ Ag bond of 3.1638 (11) $\AA$, forming a figure-of-eight-shaped complex molecule. Each silver ion is coordinated by two pyridine $\mathrm{N}$ atoms and by two carboxamide $\mathrm{O}$ atoms of neighbouring molecules, hence forming a three-dimensional framework. The nitrate anion is linked to the framework by $\mathrm{N}-\mathrm{H} \cdots \mathrm{O}$ and $\mathrm{C}-\mathrm{H} \cdots \mathrm{O}$ hydrogen bonds.

\section{Chemical context}

The title ligand, $N^{2}, N^{3}$-bis(pyridin-4-ylmethyl)pyrazine-2,3dicarboxamide (L1), is one of a series of ligands synthesized in order to study the superexchange in supramolecular complexes formed using pyrazine carboxamide derivatives and first row transition metal ions (Cati, 2002; Cati et al., 2004). To the best of our knowledge, neither the synthesis nor the crystal structure of (L1) have been described previously. It is very similar to the ligand $N^{2}, N^{3}$-bis(pyridin-2-ylmethyl)pyrazine-2,3-dicarboxamide (L2), for which a number of transition metal complexes have been described, including some interesting tetranuclear $2 \times 2$ grid-like and square complexes (Hausmann et al., 2003; Klingele et al., 2007). Two such complexes, $\left\{\left[\mathrm{Cu}_{4}(\mathbf{L 2})_{4}\right]\left(\mathrm{ClO}_{4}\right)_{4}\right\} \cdot 5 \mathrm{CH}_{3} \mathrm{OH} \cdot 4 \mathrm{H}_{2} \mathrm{O}$ and $\left\{\left[\mathrm{Ni}_{4}(\mathbf{L 2})_{4}\right] \mathrm{Cl}_{4}\right\} \cdot 5 \mathrm{CH}_{3} \mathrm{CN} \cdot 13 \mathrm{H}_{2} \mathrm{O}$ (Cati et al., 2004), exhibit 
anion encapsulation, and magnetic susceptibility measurements indicate that they are weakly anti-ferromagnetic, with $J$ values of -5.87 and $-2.64 \mathrm{~cm}^{-1}$, respectively.

A search of the Cambridge Structural Database (CSD; Groom et al., 2016), indicated that silver nitrate is an excellent metal salt for the formation of multi-dimensional coordination polymers. The silver ion can have multiple coordination geometries and modes, and the nitrate anion has been shown to coordinate to metal ions in a number of different modes, many of which involve bridging metal ions. The properties of the complexes formed are extremely varied. For example, with the tetradendate ligand 1,6-bis( $2 H$-1,2,3-triazol-2-yl)hexane, Huo et al. (2016) synthesized the three-dimensional coordination polymer, catena-[ $\left[\mu-2,2^{\prime}\right.$-(butane-1,4-diyl)bis $(2 H-1,2,3-$ triazole)]bis( $\mu$-nitrato)disilver]. They showed that it exhibits highly selective and sensitive luminescence sensing of $\mathrm{Cr}_{2} \mathrm{O}_{7}{ }^{2-}$ ions in aqueous solution. With the rigid tripodal arene-corebased nitrogen ligand, 1,3,5-tris(pyrazol-1-yl)benzene, Shu et al. (2006) formed a porous metal-organic framework, viz. catena-[bis $\left(\mu_{3}\right.$-nitrato- $\left.O, O^{\prime}, O^{\prime \prime}\right)$ bis $\left(\mu_{3}-1,3,5\right.$-tris(pyrazol-1-yl)benzene- $\left.N, N^{\prime}, N^{\prime \prime}\right)$ trisilver(I) nitrate]. The nitrate counteranions located in the cationic framework can be exchanged reversibly without destruction of the structure. Hence, this compound can act as a zeolite-like porous material for anion exchange.

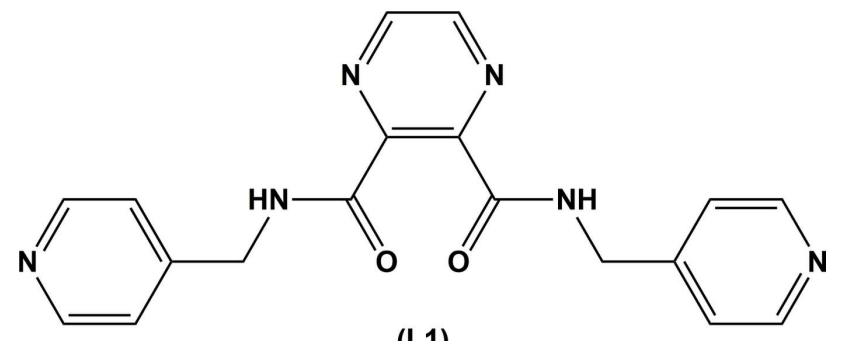

(L1)

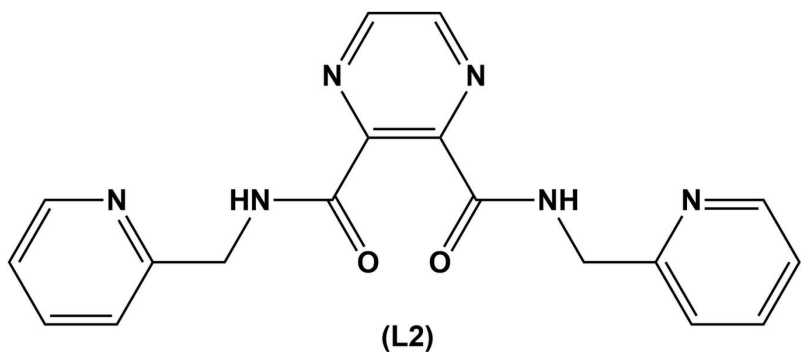

The title ligand has potentially two bidentate $(\mathrm{N}, \mathrm{N})$ and two monodentate $\left(\mathrm{N}_{\text {pyridine }}\right)$ coordination sites. It is therefore an interesting ligand to study its coordination behaviour with silver nitrate, and herein, we describe the solid state structures of ligand (L1), and the new three-dimensional coordination polymer, poly $\left[\left[\left[\mu_{4}-N^{2}, N^{3}\right.\right.\right.$-bis(pyridin-4-ylmethyl)pyrazine2,3-dicarboxamide]silver(I)]nitrate] (I).

\section{Structural commentary}

The title ligand (L1) crystallized as a dihydrate, and its molecular structure is illustrated in Fig. 1. The molecule is U- shaped with the carboxamide groups $(\mathrm{C} 6 / \mathrm{N} 3 / \mathrm{C} 5 / \mathrm{O} 1)$ being cis to one another, making a dihedral angle of $81.6(5)^{\circ}$. The terminal pyridine rings $(\mathrm{N} 4 / \mathrm{C} 7-\mathrm{C} 11)$ are inclined to one another by $58.5(4)^{\circ}$. There is an intramolecular $\mathrm{N}-\mathrm{H} \cdots \mathrm{N}$ hydrogen bond present, forming an $S(5)$ ring motif (Fig. 1 and Table 1).

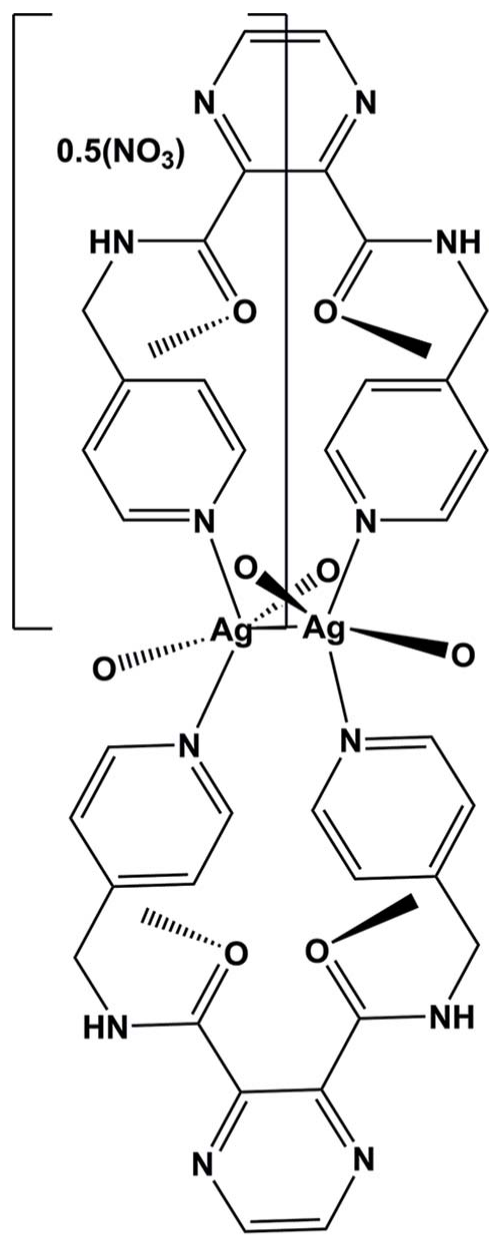

(I)

The reaction of the ligand with silver(I) nitrate led to the formation of a three-dimensional coordination polymer (I). The coordination of the ligand to the silver ions is illustrated in Fig. 2. Selected bond lengths and angles in (I) are given in Table 2. The asymmetric unit is composed of a silver ion, located on a twofold rotation axis, half a ligand molecule and half a nitrate anion. The full molecule of the ligand is generated by twofold rotational symmetry, with this twofold axis bisecting the $\mathrm{C} 4-\mathrm{C}^{\mathrm{i}}$ bonds of the pyrazine ring and the $\mathrm{Ag} 1-\mathrm{Ag} 11^{\mathrm{i}}$ bond (Table 2). The carboxamide groups (C6/N3/ $\mathrm{C} 5 / \mathrm{O} 1)$ are now trans to one another, making a dihedral angle of $65.8(4)^{\circ}$. The terminal pyridine rings $(\mathrm{N} 4 / \mathrm{C} 7-\mathrm{C} 11)$ are inclined to one another by $6.6(3)^{\circ}$. Two ligands effectively wrap around a $\mathrm{Ag}-\mathrm{Ag}$ bond of 3.1638 (11) $\AA$, forming a figure-of-eight-shaped molecule, with each silver ion being coordinated by two pyridine $\mathrm{N}$ atoms. The silver ions are each further coordinated by the carboxamide $\mathrm{O}$ atom, $\mathrm{O} 1$, of neighbouring molecules, hence forming a three-dimensional 


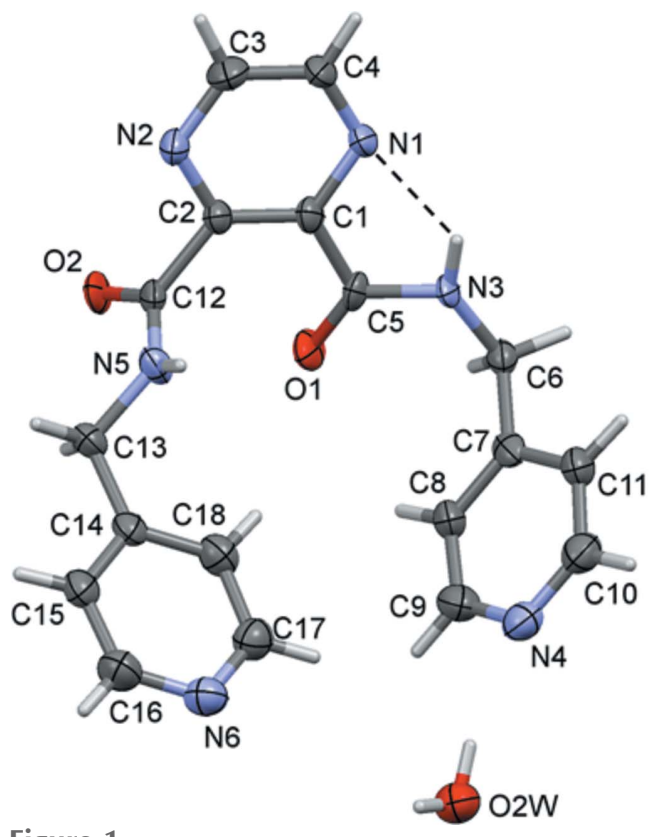

Figure 1

A view of the molecular structure of ligand (L1), with atom labelling. Displacement ellipsoids are drawn at the $50 \%$ probability level. The intramolecular $\mathrm{N}-\mathrm{H} \cdots \mathrm{N}$ contact is shown as a dashed line (see Table 2).

framework, illustrated in Fig. 3. If one considers that the silver ion, $\mathrm{Ag} 1$, is fivefold coordinate $\left(\mathrm{N}_{2} \mathrm{O}_{2} \mathrm{Ag}^{\mathrm{i}}\right)$ then its coordination sphere can be described as distorted trigonal-bipyramidal, with a $\tau_{5}$ value of $0.8\left(\tau_{5}=1\right.$ for perfect trigonalpyramidal geometry and 0 for perfect square-pyramidal geometry; Addison et al., 1984). However, if one considers the $\mathrm{Ag} 1$ ion to be fourfold coordinate, $\mathrm{N}_{2} \mathrm{O}_{2}$, with a $\tau_{4}$ value of 0.55 , its coordination sphere can be described as intermediate between trigonal-pyramidal and seesaw $\left(\tau_{4}=1\right.$ for a perfect tetrahedral geometry and 0 for a perfect square-planar geometry. For intermediate structures, including trigonalpyramidal and seesaw, $\tau_{4}$ falls within the range of 0 to 1 ; Yang et al., 2007). The nitrate anion that does not coordinate to the silver(I) ion is positionally disordered, and also located about a twofold rotation axis.
Table 1

Hydrogen-bond geometry $\left(\AA,^{\circ}\right)$ for (L1).

\begin{tabular}{lllll}
\hline$D-\mathrm{H} \cdots A$ & $D-\mathrm{H}$ & $\mathrm{H} \cdots A$ & $D \cdots A$ & $D-\mathrm{H} \cdots A$ \\
\hline $\mathrm{N} 3-\mathrm{H} 3 N \cdots \mathrm{N} 1$ & $0.88(3)$ & $2.08(5)$ & $2.718(7)$ & $129(5)$ \\
$\mathrm{N} 5-\mathrm{H} 5 N \cdots \mathrm{O} 1^{\mathrm{i}}$ & $0.88(3)$ & $2.05(4)$ & $2.858(7)$ & $151(6)$ \\
$\mathrm{O} 1 W-\mathrm{H} 1 W A \cdots \mathrm{O} 2 W^{\mathrm{ii}}$ & 0.86 & 1.91 & $2.762(7)$ & 177 \\
$\mathrm{O} 1 W-\mathrm{H} 1 W B \cdots \mathrm{N} 6^{\mathrm{iii}}$ & 0.94 & 1.97 & $2.886(7)$ & 164 \\
$\mathrm{O} 2 W-\mathrm{H} 2 W A \cdots \mathrm{O} 1 W^{\text {iv }}$ & 0.86 & 1.91 & $2.765(8)$ & 172 \\
$\mathrm{O} 2 W-\mathrm{H} 2 W B \cdots \mathrm{N} 4$ & 0.85 & 2.06 & $2.888(7)$ & 164 \\
$\mathrm{C} 3-\mathrm{H} 3 \cdots \mathrm{O} 1 W^{\mathrm{v}}$ & 0.95 & 2.38 & $3.273(8)$ & 156 \\
$\mathrm{C} 4-\mathrm{H} 4 \cdots \mathrm{O} 2 W^{\text {vi }}$ & 0.95 & 2.58 & $3.253(8)$ & 128 \\
$\mathrm{C} 6-\mathrm{H} 6 A \cdots \mathrm{N} 2^{\text {vii }}$ & 0.99 & 2.57 & $3.515(8)$ & 159 \\
$\mathrm{C} 16-\mathrm{H} 16 \cdots \mathrm{N} 4^{\text {iv }}$ & 0.95 & 2.60 & $3.451(9)$ & 149 \\
\hline
\end{tabular}

Symmetry codes: (i) $x-1, y, z$; (ii) $x,-y+1, z+\frac{1}{2}$; (iii) $x+1,-y+1, z+\frac{1}{2}$; (iv) $x-1,-y+1, z-\frac{1}{2}$; (v) $x-1,-y+2, z-\frac{1}{2}$; (vi) $x, y+1, z$; (vii) $x+1,-y+2, z+\frac{1}{2}$.

Table 2

Selected geometric parameters $\left(\AA,^{\circ}\right)$ for $(\mathbf{I})$.

\begin{tabular}{lclr}
\hline $\mathrm{Ag} 1-\mathrm{Ag} 1^{\mathrm{i}}$ & $3.1638(11)$ & $\mathrm{Ag} 1-\mathrm{O} 1^{\mathrm{ii}}$ & $2.814(5)$ \\
$\mathrm{Ag} 1-\mathrm{N} 4$ & $2.109(5)$ & & \\
& & & \\
$\mathrm{N} 4-\mathrm{Ag} 1-\mathrm{N} 4^{\mathrm{iii}}$ & $173.0(2)$ & $\mathrm{O} 1^{\mathrm{ii}}-\mathrm{Ag} 1-\mathrm{N} 4$ & $97.80(15)$ \\
$\mathrm{O} 1^{\mathrm{ii}}-\mathrm{Ag} 1-\mathrm{O} 1^{\mathrm{iv}}$ & $109.48(14)$ & $\mathrm{O} 1^{\mathrm{iv}}-\mathrm{Ag} 1-\mathrm{N} 4$ & $86.26(15)$ \\
$\mathrm{Ag} 1^{\mathrm{i}}-\mathrm{Ag} 1-\mathrm{N} 4$ & $86.51(14)$ & $\mathrm{Ag} 1^{\mathrm{i}}-\mathrm{Ag} 1-\mathrm{O} 1^{\mathrm{ii}}$ & $125.26(10)$ \\
\hline
\end{tabular}

Symmetry codes: (i) $x,-y+\frac{7}{4},-z+\frac{3}{4}$; (ii) $x-\frac{1}{4}, y+\frac{1}{4},-z+\frac{1}{2}$; (iii) $-x+\frac{7}{4}, y,-z+\frac{3}{4}$; (iv) $-x+2, y+\frac{1}{4}, z+\frac{1}{4}$.

\section{Supramolecular features}

In the crystal of ligand (L1), molecules are linked by $\mathrm{N}-$ $\mathrm{H} \cdots \mathrm{O}$ (water) hydrogen bonds forming chains propagating along the $c$-axis direction (Table 1 and Fig. 4). Parallel to this chain of molecules is a chain of hydrogen-bonded water molecules (Table 1 and Fig. 4), which is linked to the chain of (L1) molecules by $\mathrm{O}-\mathrm{H} \cdots \mathrm{N}$ hydrogen bonds, forming columns propagating along the $c$ axis. The columns are linked by $\mathrm{C}-\mathrm{H} \cdots \mathrm{O}$ and $\mathrm{C}-\mathrm{H} \cdots \mathrm{N}$ hydrogen bonds, forming a three-dimensional supramolecular structure (Table 1 and Fig. 5).

In (I), the nitrate anion is situated in the cavities of the three-dimensional framework and is linked to the framework by $\mathrm{N}-\mathrm{H} \cdots \mathrm{O}$ and $\mathrm{C}-\mathrm{H} \cdots \mathrm{O}$ hydrogen bonds (Table 3 and

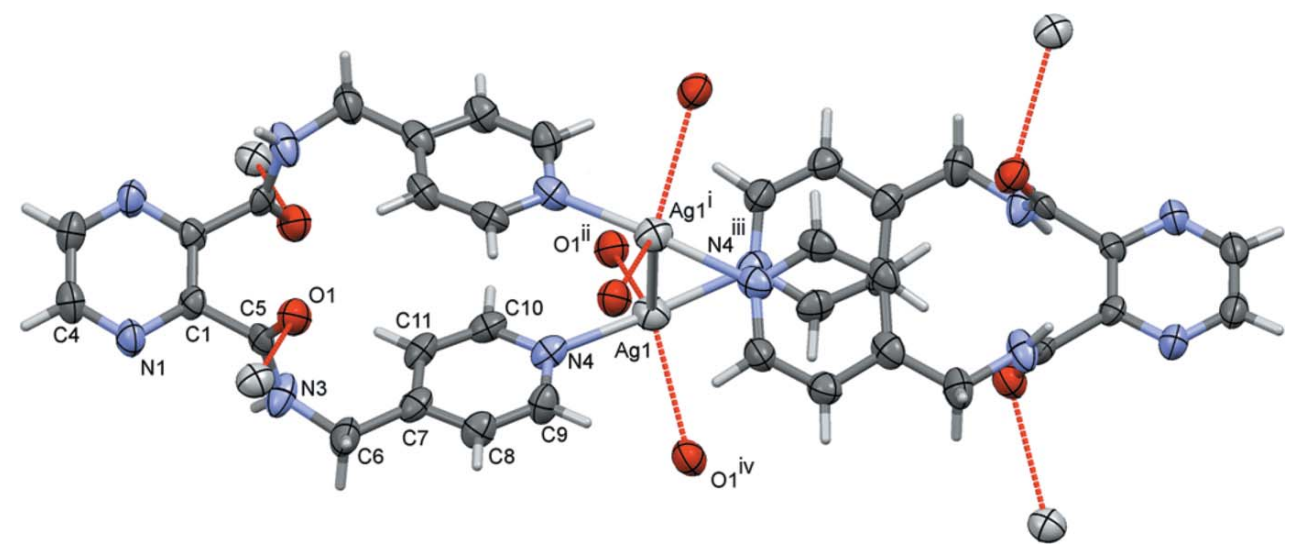

Figure 2

A view of the figure-eight arrangement of the title complex (I), with atom labelling for the asymmetric unit and some symmetry-related atoms (see Table 1 for details). The unlabelled atoms of the ligand on the left-hand-side of the figure are related to the labelled atoms by twofold rotational symmetry (symmetry operation: $-x+\frac{7}{4},-y+\frac{7}{4}, z$ ). The nitrate anions have been omitted for clarity. 


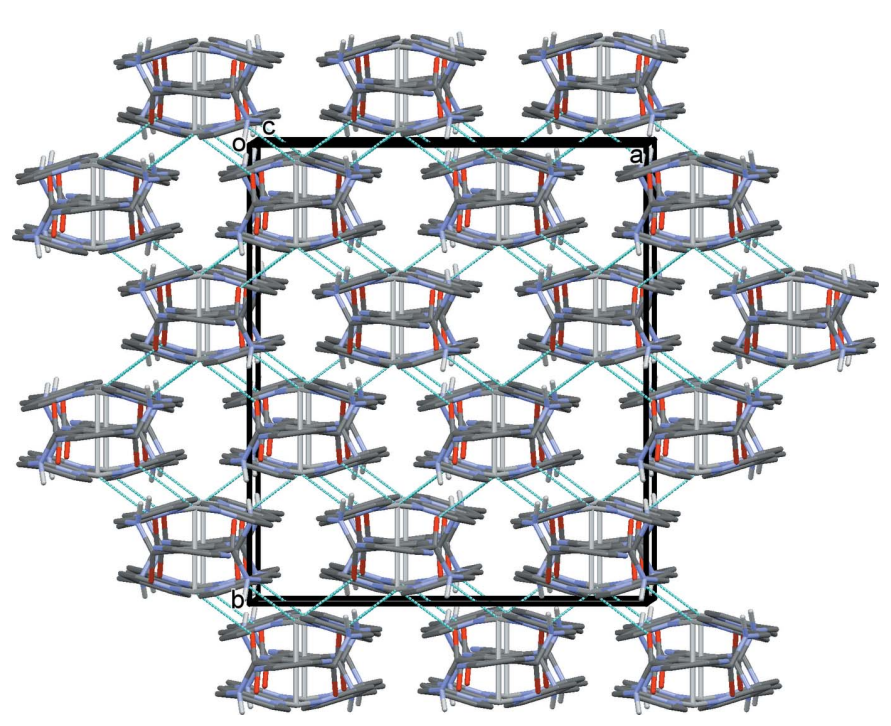

Figure 3

A view along the $c$ axis of the three-dimensional framework of complex (I), showing the $\mathrm{Ag} \cdots \mathrm{O}$ bonds as dashed lines (see Table 2). The nitrate anions and the $\mathrm{C}$-bound $\mathrm{H}$ atoms have been omitted for clarity.

Fig. 6). The nitrate anion in (I) is not essential for forming the three-dimensional structure, although it may act as a template for the formation of the framework (Batten et al., 2009). This is in contrast to the MOF catena-[bis $\left(\mu_{3}\right.$-nitrato- $\left.O, O^{\prime}, O^{\prime \prime}\right)$ bis $\left(\mu_{3}-1,3,5\right.$-tris(pyrazol-1-yl)benzene- $\left.N, N^{\prime}, N^{\prime \prime}\right) \operatorname{trisilver}(\mathrm{I})$ nitrate] mentioned above (Shu et al., 2006), in which there are nitrate anions coordinating the silver ions in a $\mu_{3}$ fashion and present also in the framework cavities. There are, of course, other examples reported in the Cambridge Structural Database (Groom et al., 2016).

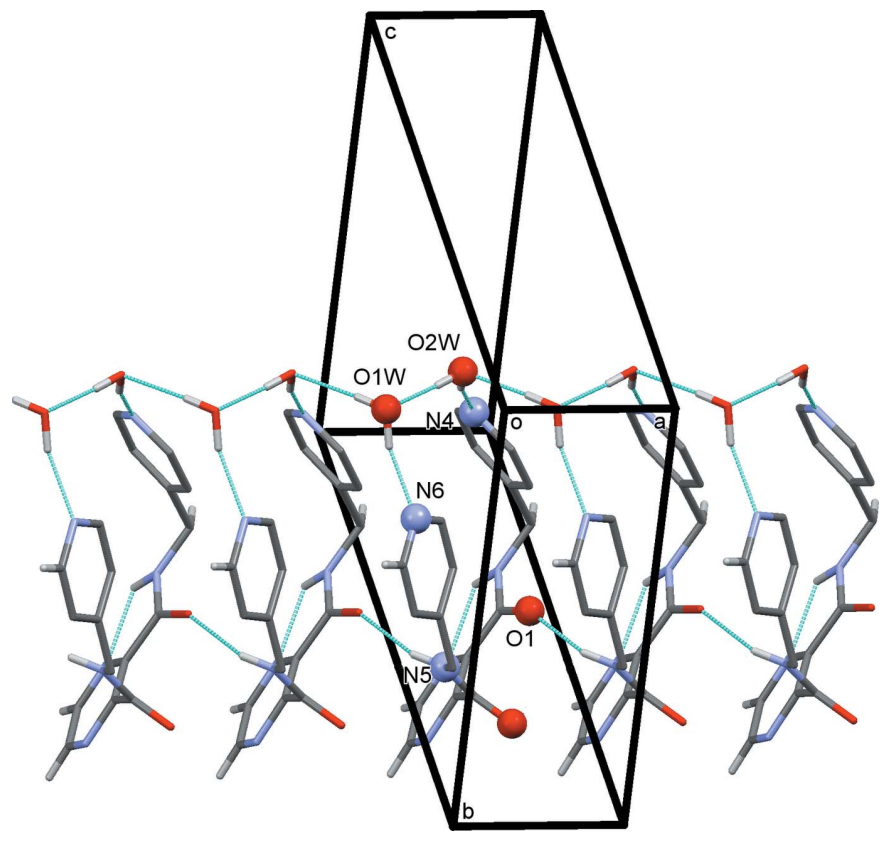

Figure 4

A partial view along direction [111] of the crystal packing of ligand (L1). The hydrogen bonds are shown as dashed lines (see Table 1)
Table 3

Hydrogen-bond geometry $\left(\AA,^{\circ}\right)$ for $(\mathbf{I})$.

\begin{tabular}{lllll}
\hline$D-\mathrm{H} \cdots A$ & $D-\mathrm{H}$ & $\mathrm{H} \cdots A$ & $D \cdots A$ & $D-\mathrm{H} \cdots A$ \\
\hline $\mathrm{N} 3-\mathrm{H} 3 N \cdots \mathrm{O} 11^{\mathrm{v}}$ & 0.88 & 1.86 & $2.744(14)$ & 178 \\
$\mathrm{~N} 3-\mathrm{H} 3 N \cdots \mathrm{O} 13^{\mathrm{v}}$ & 0.88 & 2.26 & $2.875(13)$ & 127 \\
$\mathrm{C} 4-\mathrm{H} 4 \cdots \mathrm{O} 11^{\mathrm{vi}}$ & 0.95 & 2.45 & $3.378(13)$ & 165 \\
$\mathrm{C} 4-\mathrm{H} 4 \cdots \mathrm{O} 14^{\mathrm{vii}}$ & 0.95 & 2.40 & $3.33(2)$ & 168 \\
$\mathrm{C} 9-\mathrm{H} 9 \cdots \mathrm{O} 13^{\mathrm{viii}}$ & 0.95 & 2.50 & $3.224(14)$ & 133 \\
$\mathrm{C} 11-\mathrm{H} 11 \cdots \mathrm{O} 13^{\mathrm{v}}$ & 0.95 & 2.51 & $3.154(13)$ & 126 \\
\hline
\end{tabular}

Symmetry codes: (v) $x, y+1, z$; (vi) $-x+2,-y+1,-z$; (vii) $-x+2, y+\frac{3}{4}, z-\frac{1}{4}$; (viii) $x+\frac{1}{4}, y+\frac{3}{4},-z+\frac{1}{2}$.

In describing compound (I) as a three-dimensional coordination polymer, we make here the distinction between a coordination polymer and a metal-organic framework. Both have a three-dimensional framework but there are no cavities, even small ones, in the structure of (I). Hence, it should be classed as a three-dimensional coordination polymer according to the IUPAC recommendations on the 'Terminology of metal-organic frameworks and coordination polymers' (Batten et al., 2013).

\section{Database survey}

A search of the Cambridge Structural Database (Version 5.38, update February 2017; Groom et al., 2016) for $\mathrm{Ag}-\mathrm{Ag}$ complexes, excluding silver ion clusters of any kind, gave 321 hits. Limiting the search to $\mathrm{Ag}-\mathrm{Ag}$ complexes with each silver ion coordinated by two pyridine $\mathrm{N}$ atoms, gave 95 hits. The $\mathrm{Ag}-\mathrm{Ag}$ distances vary between $\mathrm{ca}$ 2.6-3.6 $\AA$. One compound, bis[ $\mu_{2}$-2,7-di-tert-butyl-9,9-dimethyl- $N, N^{\prime}$-bis[(3-pyridyl)methyl]xanthene-4,5-dicarboxamide]disilver bis(trifluoromethanesulfonate) chloroform solvate (HIFKUD; Yue et al., 2007), is particularly interesting because it too involves a

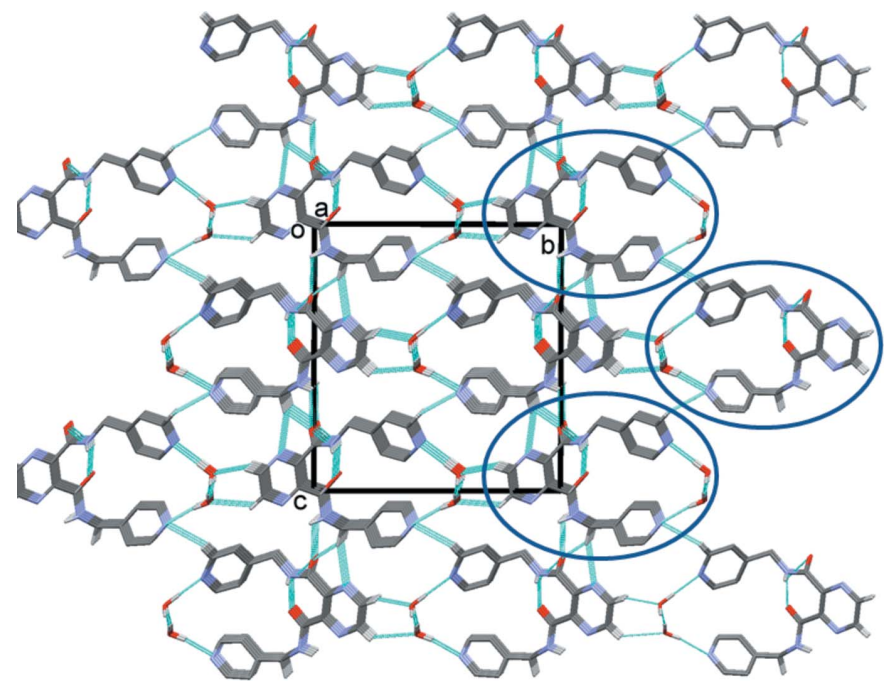

Figure 5

A view along the $a$ axis of the crystal packing of ligand (L1). The columns of (L1) molecules, linked by hydrogen bonds involving the water molecules, are indicated by blue circles. The hydrogen bonds are shown as dashed lines (see Table 1), and for clarity, only the $\mathrm{H}$ atoms involved in hydrogen bonding have been included. 


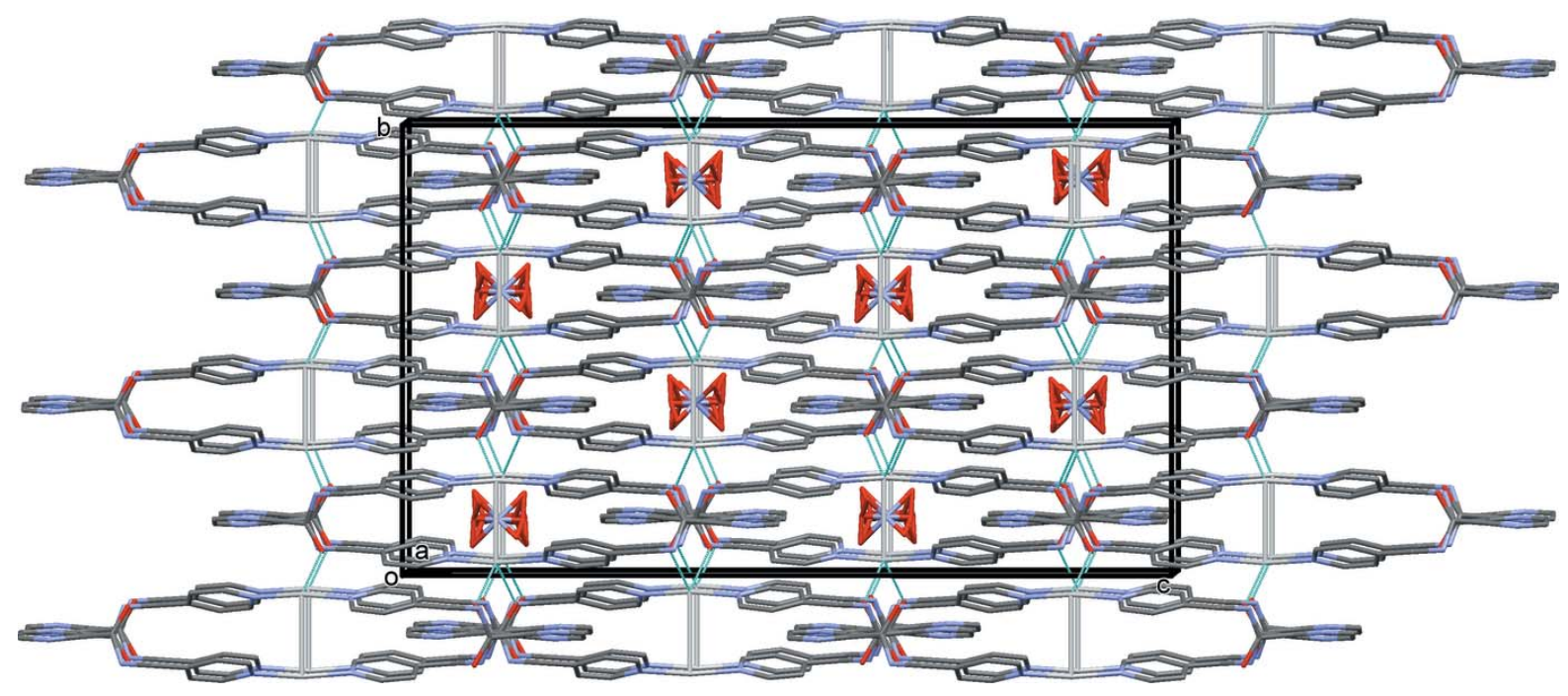

Figure 6

A view along the $a$ axis of the crystal packing of complex (I), showing the Ag $\cdots$ O bonds as dashed lines (see Table 2). For clarity, all $\mathrm{H}$ atoms have been omitted.

dicarboxamide ligand, viz. $N, N^{\prime}$-bis[(3-pyridyl)methy]xanthene-4,5-dicarboxamide), that wraps around an $\mathrm{Ag}-\mathrm{Ag}$ bond forming a similar figure-of-eight-shaped complex. Here the $\mathrm{Ag}-\mathrm{Ag}$ bond length is 3.134 (1) $\AA$, slightly shorter than the value of 3.1638 (11) $\AA$ observed in (I); Table 2. A search for the benzene analogue of ligand (L1), $N$-(4-pyridylmethyl)carbamoyl)benzene, gave only two hits. Both of them are mercury(II) complexes, viz. the binuclear complex bis $\left\{\mu_{2}-1,2-\right.$ bis[ $N$-(4-pyridylmethyl)carbamoyl]benzene)tetrakis(trifluoroacetato)dimercury(II) methanol solvate (XAHSIJ; Burchell et al., 2004) and the two-dimensional network catena-[bis $\left\{\mu_{2^{-}}\right.$ 1,2-bis [ $N$-(4-pyridylmethyl)carbamoyl]benzene \}dichloridomercury(II) 1,2-dichloroethane solvate] (XAHSOP; Burchell et al., 2004). A search for the benzene analogue of ligand (L2), [ $N$-(2-pyridylmethyl)carbamoyl]benzene, gave zero hits, while that for [ $\mathrm{N}$-(3-pyridylmethyl)carbamoyl]benzene gave eight hits. The latter includes the crystal structure of the dihydrate of the ligand itself (PANROM; Ge et al., 2005) and the structures of seven first-row transition metal one-, two- and three-dimensional coordination polymers.

\section{Synthesis and crystallization}

Ligand (L1) was prepared using the same procedure as for ligand (L2) (Cati et al., 2004). Dimethyl pyrazine-2,3-dicarboxylate (1.96 g, $10 \mathrm{mmol}$; Alvarez-Ibarra et al., 1994) and an excess of 4 -(aminomethyl)pyridine $(3.24 \mathrm{~g}, 30 \mathrm{mmol})$ in $35 \mathrm{ml}$ of methanol were heated to reflux and heating was continued for $72 \mathrm{~h}$ in a two-necked flask $(100 \mathrm{ml})$. The brown solution that formed was concentrated and $15 \mathrm{ml}$ of water were added, which precipitated quantitatively ligand $(\mathbf{L 1})$. The solid was collected by filtration, washed with $10 \mathrm{ml}$ of water and dried in air. Recrystallization in ethanol gave colourless plate-like crystals (yield is quantitative; m.p. $474 \mathrm{~K}$ ). Spectroscopic data: ${ }^{1} \mathrm{H}$ NMR (400 MHz, DMSO-d6): $9.33(t, 1 \mathrm{H}$,
$\left.J_{\mathrm{hg}}=6.1, \mathrm{Hh}\right) ; 8.86(s, 1 \mathrm{H}, \mathrm{H} n=\mathrm{H} m) ; 8.49\left(d d, 2 \mathrm{H}, J_{\mathrm{ba}}=4.5\right.$, $\left.J_{\mathrm{be}}=1.5, \mathrm{H} b=\mathrm{H} d\right) ; 7.39\left(d d, 2 \mathrm{H}, J_{\mathrm{ab}}=4.5, J_{\mathrm{eb}}=1.5, \mathrm{H} a=\mathrm{H} e\right)$; $4.52\left(d, 2 \mathrm{H}, J_{\mathrm{gh}}=6.1, \mathrm{Hg}\right) .{ }^{13} \mathrm{C}$ NMR $(400 \mathrm{MHz}, \mathrm{DMSO}-d 6)$ : 165.8, 150.3, 148.9, 147.6, 145.6, 123.0, 42.2. IR (KBr pellet, $\left.\mathrm{cm}^{-1}\right)$ : $3273(s), 3031(s), 1675(v s), 1602(v s), 1564(v s), 1520$ $(v s), 1416(v s), 1364(s), 1311(s), 1292(s), 1220(s), 1185(m)$, $1164(\mathrm{~m}), 1124(\mathrm{~s}), 1069(\mathrm{~m}), 995(\mathrm{~s}), 871(\mathrm{w}), 830(\mathrm{~m}), 787(\mathrm{~m})$, $745(\mathrm{~m}), 715(\mathrm{~m}), 611(\mathrm{~m}), 575(\mathrm{w}), 504(\mathrm{~m}), 495(\mathrm{~m}), 475(\mathrm{~m})$. Elemental analysis for $\left[\mathrm{C}_{18} \mathrm{H}_{16} \mathrm{~N}_{6} \mathrm{O}_{2}\right] \cdot \mathrm{H}_{2} \mathrm{O} \quad\left(M_{\mathrm{r}}=\right.$ $366.39 \mathrm{~g} \mathrm{~mol}^{-1}$ ): calculated: C: $59.01 \mathrm{H}: 4.95 \mathrm{~N}: 22.94 \%$; found: C: 59.10 H: $5.05 \mathrm{~N}: 23.10 \%$.

Complex (I): A solution of (L1) (46 mg; $0.126 \mathrm{mmol})$ in $6 \mathrm{ml}$ $\mathrm{CHCl}_{3}$ was introduced into a $13 \mathrm{~mm}$ diameter glass tube. It was layered with methanol ( $c a 2 \mathrm{ml})$ used as a buffer zone. A solution of $\mathrm{AgNO}_{3}(21 \mathrm{mg}, 0.126 \mathrm{mmol})$ in $\mathrm{MeOH}(6 \mathrm{ml})$ was then added gently to avoid possible mixing. The glass tube was sealed with a perforated parafilm and left at room temperature. Colourless block-like crystals were obtained after a few days (yield $60 \mathrm{mg}$, 92\%). Elemental analysis for $\mathrm{AgC}_{18} \mathrm{H}_{16} \mathrm{~N}_{7} \mathrm{O}_{5}:\left(M_{\mathrm{r}}=518.25 \mathrm{~g} \mathrm{~mol}^{-1}\right)$ : calculated: $\mathrm{C}: 41.72 \mathrm{H}$ : $3.11 \mathrm{~N}: 18.92 \%$; found: C: $41.65 \mathrm{H}: 3.09 \mathrm{~N}: 18.85 \%$.

\section{Refinement}

Crystal data, data collection and structure refinement details are summarized in Table 4. For the ligand (L1), the NH and water $\mathrm{H}$ atoms were located in difference-Fourier maps and refined with distance restraints: $\mathrm{O}-\mathrm{H}=0.85$ (2) $\AA$, $\mathrm{N}-\mathrm{H}=$ $0.88(2) \AA$ with $U_{\text {iso }}(\mathrm{H})=1.5 U_{\text {eq }}(\mathrm{O})$ and $1.2 U_{\text {eq }}(\mathrm{N})$. In the final cycles of refinement, the water $\mathrm{H}$ atoms were treated as riding atoms. For complex (I), the NH H atoms were included in calculated positions and treated as riding: $\mathrm{N}-\mathrm{H}=0.88 \AA$ with $U_{\text {iso }}(\mathrm{H})=1.2 U_{\text {eq }}(\mathrm{N})$. For both compounds, the C-bound $\mathrm{H}$ atoms were included in calculated positions and refined as riding: $\mathrm{C}-\mathrm{H}=0.95-0.99 \AA$ with $U_{\text {iso }}(\mathrm{H})=1.2 U_{\text {eq }}(\mathrm{C})$. The 
Table 4

Experimental details.

(L1)

Crystal data

Chemical formula

$M_{\mathrm{r}}$

Crystal system, space group

Temperature (K)

$a, b, c(\AA)$

$\alpha, \beta, \gamma\left({ }^{\circ}\right)$

$V\left(\AA^{3}\right)$

Z

Radiation type

$\mu\left(\mathrm{mm}^{-1}\right)$

Crystal size (mm)

Data collection

Diffractometer

Absorption correction

$T_{\min }, T_{\max }$

No. of measured, independent and

observed $[I>2 \sigma(I)]$ reflections

$R_{\text {int }}$

$(\sin \theta / \lambda)_{\max }\left(\AA^{-1}\right)$

Refinement

$R\left[F^{2}>2 \sigma\left(F^{2}\right)\right], w R\left(F^{2}\right), S$

No. of reflections

No. of parameters

No. of restraints

$\mathrm{H}$-atom treatment

$\Delta \rho_{\max }, \Delta \rho_{\min }\left(\mathrm{e} \AA^{-3}\right)$

\author{
$\mathrm{C}_{18} \mathrm{H}_{16} \mathrm{~N}_{6} \mathrm{O}_{2} \cdot 2 \mathrm{H}_{2} \mathrm{O}$ \\ 384.40 \\ Monoclinic, $P c$ \\ 153 \\ 4.3677 (6), 14.0232 (12), 15.1816 (18) \\ $90,96.153$ (16), 90 \\ $924.50(19)$ \\ 2 \\ Mo $K \alpha$ \\ 0.10 \\ $0.50 \times 0.15 \times 0.05$
}

(I)

$\left[\mathrm{Ag}\left(\mathrm{C}_{18} \mathrm{H}_{16} \mathrm{~N}_{6} \mathrm{O}_{2}\right)\right] \mathrm{NO}_{3}$
518.25
Orthorhombic, $F d d d$
153
$14.9776(16), 17.3228(12), 29.570(4)$
$90,90,90$
$7672.1(14)$
16
$\mathrm{Mo} \mathrm{K \alpha}$
1.10
$0.40 \times 0.30 \times 0.20$

Stoe IPDS 1

Multi-scan (MULABS; Spek, 2009)

$0.763,1.000$

$7134,3388,1693$

Stoe IPDS 1

Multi-scan (MULABS; Spek, 2009)

$0.985,1.000$

$13548,1721,1038$

0.107

0.615

0.096

0.600

$0.051,0.122,0.77$
3388
260
8
$\mathrm{H}$ atoms treated by a mixture of independent
$\quad$ and constrained refinement
$0.21,-0.25$

$0.045,0.107,0.90$

1721

141

0

$\mathrm{H}$-atom parameters constrained

$0.68,-0.65$

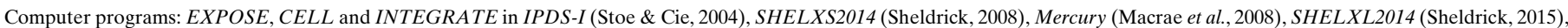
PLATON (Spek, 2009) and publCIF (Westrip, 2010).

nitrate anion is positionally disordered about a twofold rotation axis and was refined with fixed occupancies (N10A and $\mathrm{N} 10 B=0.5, \mathrm{O} 11$ and $\mathrm{O} 13=0.5, \mathrm{O} 12$ and $\mathrm{O} 14=0.25)$, and all their ADP's were made equal to that of atom O11. Using a one-circle image-plate diffraction system it is not possible to measure $100 \%$ of the Ewald sphere, particularly for triclinic or monoclinic systems. This is the case for ligand (L1), which crystallized in the monoclinic space group $P c$ and for which only $94.7 \%$ of the Ewald sphere was accessible.

\section{Funding information}

Funding for this research was provided by: Swiss National Science Foundation; University of Neuchâtel..

\section{References}

Addison, A. W., Rao, T. N., Reedijk, J., van Rijn, J. \& Verschoor, G. C. (1984). J. Chem. Soc. Dalton Trans. pp. 1349-1356.

Alvarez-Ibarra, C., Cuervo-Rodríguez, R., Fernández-Monreal, M. C. \& Ruiz, M. P. (1994). J. Org. Chem. 59, 7284-7291.

Batten, S. R., Champness, N. R., Chen, X. M., Garcia-Martinez, J., Kitagawa, S., Öhrström, L., O'Keeffe, M., Suh, M. P. \& Reedijk, J. (2013). Pure Appl. Chem. 5, 1715-1724.

Batten, S. R., Neville, S. M. \& Turner, D. R. (2009). Coordination Polymers, Design, Analysis and Applications. Cambridge: Royal Society of Chemistry.
Burchell, T. J., Eisler, D. J. \& Puddephatt, R. J. (2004). Inorg. Chem. 43, 5550-5557.

Cati, D. (2002). PhD thesis, University of Neuchâtel, Switzerland.

Cati, D. S., Ribas, J., Ribas-Ariño, J. \& Stoeckli-Evans, H. (2004). Inorg. Chem. 43, 1021-1030.

Ge, C.-H., Kou, H.-Z., Wang, R.-J., Jiang, Y.-B. \& Cui, A.-L. (2005). Acta Cryst. E61, o2024-o2026.

Groom, C. R., Bruno, I. J., Lightfoot, M. P. \& Ward, S. C. (2016). Acta Cryst. B72, 171-179.

Hausmann, J., Jameson, G. B. \& Brooker, S. (2003). Chem. Commun. pp. 2992-2993.

Huo, J. Z., Su, X. M., Wu, X. X., Liu, Y. Y. \& Ding, B. (2016). CrystEngComm, 18, 6640-6652.

Klingele (née Hausmann), J., Boas, J. F., Pilbrow, J. R., Moubaraki, B., Murray, K. S., Berry, K. J., Hunter, K. A., Jameson, G. B., Boyd, P. D. W. \& Brooker, S. (2007). Dalton Trans. pp. 633-645.

Macrae, C. F., Bruno, I. J., Chisholm, J. A., Edgington, P. R., McCabe, P., Pidcock, E., Rodriguez-Monge, L., Taylor, R., van de Streek, J. \& Wood, P. A. (2008). J. Appl. Cryst. 41, 466-470.

Sheldrick, G. M. (2008). Acta Cryst. A64, 112-122.

Sheldrick, G. M. (2015). Acta Cryst. C71, 3-8.

Shu, M., Tu, C., Xu, W., Jin, H. \& Sun, J. (2006). Cryst. Growth Des. 6, $1890-1896$.

Spek, A. L. (2009). Acta Cryst. D65, 148-155.

Stoe \& Cie (2004). IPDSI Bedienungshandbuch. Stoe \& Cie GmbH, Darmstadt, Germany.

Westrip, S. P. (2010). J. Appl. Cryst. 43, 920-925.

Yang, L., Powell, D. R. \& Houser, R. P. (2007). Dalton Trans. pp. $955-$ 964.

Yue, N. L. S., Jennings, M. C. \& Puddephatt, R. J. (2007). Eur. J. Inorg. Chem. pp. 1690-1697. 


\section{supporting information}

Acta Cryst. (2017). E73, 798-803 [https://doi.org/10.1107/S2056989017006387]

\section{Crystal structure of a pyrazine-2,3-dicarboxamide ligand and of its silver(I) nitrate complex, a three-dimensional coordination polymer}

\section{Dilovan S. Cati and Helen Stoeckli-Evans}

\section{Computing details}

For both compounds, data collection: EXPOSE in IPDS-I (Stoe \& Cie, 2004); cell refinement: CELL in IPDS-I (Stoe \& Cie, 2004); data reduction: INTEGRATE in IPDS-I (Stoe \& Cie, 2004); program(s) used to solve structure: SHELXS2014 (Sheldrick, 2008); program(s) used to refine structure: SHELXL2014 (Sheldrick, 2015); molecular graphics: Mercury (Macrae et al., 2008); software used to prepare material for publication: SHELXL2014 (Sheldrick, 2015), PLATON (Spek, 2009) and publCIF (Westrip, 2010).

(L1) $N^{2}, N^{3}$-Bis(pyridin-4-ylmethyl) pyrazine-2,3-dicarboxamide

Crystal data

$\mathrm{C}_{18} \mathrm{H}_{16} \mathrm{~N}_{6} \mathrm{O}_{2} \cdot 2 \mathrm{H}_{2} \mathrm{O}$

$M_{r}=384.40$

Monoclinic, $P c$

$a=4.3677(6) \AA$

$b=14.0232(12) \AA$

$c=15.1816(18) \AA$

$\beta=96.153(16)^{\circ}$

$V=924.50(19) \AA^{3}$

$Z=2$

Data collection

Stoe IPDS 1 diffractometer

Radiation source: fine-focus sealed tube

Plane graphite monochromator

$\varphi$ rotation scans

Absorption correction: multi-scan

(MULABS; Spek, 2009)

$T_{\text {min }}=0.763, T_{\text {max }}=1.000$

Refinement

Refinement on $F^{2}$

Least-squares matrix: full

$R\left[F^{2}>2 \sigma\left(F^{2}\right)\right]=0.051$

$w R\left(F^{2}\right)=0.122$

$S=0.77$

3388 reflections

260 parameters

8 restraints
$F(000)=404$

$D_{\mathrm{x}}=1.381 \mathrm{Mg} \mathrm{m}^{-3}$

Mo $K \alpha$ radiation, $\lambda=0.71073 \AA$

Cell parameters from 3264 reflections

$\theta=2.0-25.9^{\circ}$

$\mu=0.10 \mathrm{~mm}^{-1}$

$T=153 \mathrm{~K}$

Plate, colourless

$0.50 \times 0.15 \times 0.05 \mathrm{~mm}$

7134 measured reflections

3388 independent reflections

1693 reflections with $I>2 \sigma(I)$

$R_{\text {int }}=0.107$

$\theta_{\text {max }}=25.9^{\circ}, \theta_{\min }=2.0^{\circ}$

$h=-5 \rightarrow 5$

$k=-17 \rightarrow 16$

$l=-18 \rightarrow 18$

Primary atom site location: structure-invariant direct methods

Secondary atom site location: difference Fourier map

Hydrogen site location: mixed

$\mathrm{H}$ atoms treated by a mixture of independent and constrained refinement

$w=1 /\left[\sigma^{2}\left(F_{\mathrm{o}}{ }^{2}\right)+(0.0453 P)^{2}\right]$

where $P=\left(F_{\mathrm{o}}^{2}+2 F_{\mathrm{c}}^{2}\right) / 3$ 
$(\Delta / \sigma)_{\max }<0.001$

$\Delta \rho_{\max }=0.21 \mathrm{e} \AA^{-3}$

$\Delta \rho_{\min }=-0.25$ e $\AA^{-3}$
Extinction correction: (SHELXL2016; Sheldrick, 2015),

$\mathrm{Fc}^{*}=\mathrm{kFc}\left[1+0.001 \mathrm{xFc}^{2} \lambda^{3} / \sin (2 \theta)\right]^{-1 / 4}$

Extinction coefficient: $0.036(6)$

\section{Special details}

Geometry. All esds (except the esd in the dihedral angle between two 1.s. planes) are estimated using the full covariance matrix. The cell esds are taken into account individually in the estimation of esds in distances, angles and torsion angles; correlations between esds in cell parameters are only used when they are defined by crystal symmetry. An approximate (isotropic) treatment of cell esds is used for estimating esds involving l.s. planes.

Fractional atomic coordinates and isotropic or equivalent isotropic displacement parameters $\left(\AA^{2}\right)$

\begin{tabular}{|c|c|c|c|c|}
\hline & $x$ & $y$ & $z$ & $U_{\text {iso }} * / U_{\text {eq }}$ \\
\hline O1 & $0.7750(11)$ & $0.9108(3)$ & $0.4491(3)$ & $0.0358(13)$ \\
\hline $\mathrm{O} 2$ & $0.5478(11)$ & $0.9994(3)$ & $0.2578(3)$ & $0.0351(12)$ \\
\hline N1 & $0.4263(14)$ & $1.1135(3)$ & $0.5363(4)$ & $0.0321(15)$ \\
\hline $\mathrm{N} 2$ & $0.1716(14)$ & $1.1331(4)$ & $0.3588(4)$ & $0.0330(15)$ \\
\hline N3 & $0.7147(13)$ & $0.9491(3)$ & $0.5921(3)$ & $0.0258(14)$ \\
\hline $\mathrm{H} 3 \mathrm{~N}$ & $0.612(13)$ & $0.998(3)$ & $0.609(4)$ & $0.031^{*}$ \\
\hline N4 & $0.4923(14)$ & $0.5966(4)$ & $0.6225(4)$ & $0.0403(16)$ \\
\hline N5 & $0.1699(14)$ & 0.9119 (4) & $0.3107(4)$ & $0.0313(14)$ \\
\hline $\mathrm{H} 5 \mathrm{~N}$ & $0.002(11)$ & $0.906(5)$ & $0.337(4)$ & $0.038^{*}$ \\
\hline N6 & $-0.0999(16)$ & $0.5615(4)$ & $0.3180(4)$ & $0.0436(17)$ \\
\hline $\mathrm{C} 1$ & $0.4668(15)$ & $1.0457(4)$ & $0.4743(4)$ & $0.0267(17)$ \\
\hline $\mathrm{C} 2$ & $0.3401(16)$ & $1.0564(4)$ & $0.3878(4)$ & $0.0258(16)$ \\
\hline $\mathrm{C} 3$ & $0.1396(19)$ & $1.1999(5)$ & $0.4209(5)$ & $0.043(2)$ \\
\hline $\mathrm{H} 3$ & 0.030625 & 1.256737 & 0.403978 & $0.051^{*}$ \\
\hline $\mathrm{C} 4$ & $0.2586(18)$ & $1.1888(4)$ & $0.5079(5)$ & $0.0360(19)$ \\
\hline H4 & 0.219526 & 1.236935 & 0.549295 & $0.043^{*}$ \\
\hline $\mathrm{C} 5$ & $0.6613(15)$ & $0.9624(4)$ & $0.5039(4)$ & $0.0280(16)$ \\
\hline C6 & $0.9192(16)$ & $0.8748(4)$ & $0.6312(5)$ & $0.0290(17)$ \\
\hline H6A & 0.985533 & 0.891453 & 0.693707 & $0.035^{*}$ \\
\hline H6B & 1.105193 & 0.872221 & 0.599314 & $0.035^{*}$ \\
\hline $\mathrm{C} 7$ & $0.7688(16)$ & $0.7778(4)$ & $0.6278(5)$ & $0.0307(17)$ \\
\hline $\mathrm{C} 8$ & $0.8016(18)$ & $0.7151(4)$ & $0.5602(5)$ & $0.0376(19)$ \\
\hline H8 & 0.917205 & 0.733033 & 0.513344 & $0.045^{*}$ \\
\hline C9 & $0.669(2)$ & $0.6270(5)$ & $0.5600(5)$ & $0.046(2)$ \\
\hline H9 & 0.702062 & 0.584555 & 0.513239 & $0.056^{*}$ \\
\hline $\mathrm{C} 10$ & $0.4698(19)$ & $0.6573(5)$ & $0.6889(5)$ & $0.044(2)$ \\
\hline H10 & 0.357252 & 0.636975 & 0.735668 & $0.053^{*}$ \\
\hline C11 & $0.5967(17)$ & $0.7473(5)$ & $0.6950(5)$ & $0.0374(19)$ \\
\hline H11 & 0.568042 & 0.787610 & 0.743743 & $0.045^{*}$ \\
\hline $\mathrm{C} 12$ & $0.3639(17)$ & 0.9848 (4) & $0.3138(4)$ & $0.0297(17)$ \\
\hline $\mathrm{C} 13$ & $0.1367(17)$ & 0.8444 (4) & $0.2372(5)$ & $0.0337(18)$ \\
\hline $\mathrm{H} 13 \mathrm{~A}$ & 0.332934 & 0.841179 & 0.210178 & $0.040^{*}$ \\
\hline H13B & -0.024426 & 0.867753 & 0.191430 & $0.040^{*}$ \\
\hline C14 & $0.0529(17)$ & $0.7469(4)$ & $0.2655(4)$ & $0.0303(17)$ \\
\hline $\mathrm{C} 15$ & $-0.1501(17)$ & $0.6908(5)$ & $0.2141(5)$ & $0.0362(18)$ \\
\hline
\end{tabular}




$\begin{array}{lllll}\text { H15 } & -0.242090 & 0.714271 & 0.158778 & 0.043^{*} \\ \text { C16 } & -0.224(2) & 0.6001(5) & 0.2413(5) & 0.049(2) \\ \text { H16 } & -0.368187 & 0.563552 & 0.204091 & 0.058^{*} \\ \text { C17 } & 0.1048(19) & 0.6151(5) & 0.3680(6) & 0.042(2) \\ \text { H17 } & 0.200289 & 0.589113 & 0.421904 & 0.050^{*} \\ \text { C18 } & 0.1843(19) & 0.7075(5) & 0.3446(5) & 0.0389(18) \\ \text { H18 } & 0.327922 & 0.743333 & 0.382699 & 0.047^{*} \\ \text { O1W } & 0.7645(12) & 0.5986(3) & 0.9268(3) & 0.0503(15) \\ \text { H1WA } & 0.624209 & 0.595327 & 0.962023 & 0.075^{*} \\ \text { H1WB } & 0.768245 & 0.545186 & 0.889768 & 0.075^{*} \\ \text { O2W } & 0.3065(12) & 0.4183(3) & 0.5379(3) & 0.0478(14) \\ \text { H2WA } & 0.133161 & 0.418283 & 0.504471 & 0.072^{*} \\ \text { H2WB } & 0.327355 & 0.474826 & 0.557396 & 0.072^{*}\end{array}$

Atomic displacement parameters $\left(\AA^{2}\right)$

\begin{tabular}{|c|c|c|c|c|c|c|}
\hline & $U^{11}$ & $U^{22}$ & $U^{33}$ & $U^{12}$ & $U^{13}$ & $U^{23}$ \\
\hline O1 & $0.051(3)$ & $0.039(3)$ & $0.021(3)$ & $0.009(2)$ & 0.019 (3) & $0.000(2)$ \\
\hline $\mathrm{O} 2$ & $0.052(3)$ & $0.040(2)$ & 0.015 & $-0.005(2)$ & $0.015(2)$ & -0.0003 (19) \\
\hline N1 & $0.055(4)$ & $0.024(3)$ & $0.018(3)$ & $0.001(3)$ & 0.007 (3) & $-0.002(2)$ \\
\hline $\mathrm{N} 2$ & $0.050(4)$ & $0.030(3)$ & $0.019(3)$ & $0.006(3)$ & $0.004(3)$ & $0.003(2)$ \\
\hline N3 & $0.041(4)$ & $0.025(3)$ & $0.012(3)$ & $0.004(3)$ & $0.009(3)$ & $-0.001(2)$ \\
\hline N4 & $0.047(4)$ & $0.032(3)$ & $0.041(4)$ & $0.001(3)$ & $0.001(4)$ & 0.007 (3) \\
\hline N5 & $0.038(4)$ & $0.034(3)$ & $0.023(3)$ & $-0.010(3)$ & $0.006(3)$ & $-0.006(2)$ \\
\hline N6 & $0.054(4)$ & $0.033(3)$ & $0.044(4)$ & $-0.005(3)$ & 0.007 (4) & $-0.003(3)$ \\
\hline $\mathrm{C} 1$ & $0.035(5)$ & $0.029(3)$ & $0.018(4)$ & $-0.002(3)$ & $0.009(3)$ & $0.001(3)$ \\
\hline $\mathrm{C} 2$ & $0.034(4)$ & $0.025(3)$ & $0.020(4)$ & $0.000(3)$ & $0.008(3)$ & $0.000(3)$ \\
\hline $\mathrm{C} 3$ & $0.061(6)$ & $0.027(4)$ & $0.040(5)$ & $0.011(4)$ & $0.003(4)$ & $0.003(3)$ \\
\hline $\mathrm{C} 4$ & $0.060(5)$ & $0.025(4)$ & $0.023(4)$ & $0.007(4)$ & $0.009(4)$ & $-0.003(3)$ \\
\hline $\mathrm{C} 5$ & $0.037(4)$ & $0.031(4)$ & $0.017(4)$ & $-0.001(3)$ & $0.006(3)$ & $0.008(3)$ \\
\hline C6 & $0.036(4)$ & $0.031(4)$ & $0.020(4)$ & $0.006(3)$ & $0.001(3)$ & $0.000(3)$ \\
\hline $\mathrm{C} 7$ & $0.032(5)$ & $0.030(4)$ & $0.027(4)$ & $0.013(3)$ & -0.007 (4) & $-0.002(3)$ \\
\hline $\mathrm{C} 8$ & $0.056(5)$ & $0.034(4)$ & $0.022(5)$ & $0.001(4)$ & $0.001(4)$ & $-0.001(3)$ \\
\hline $\mathrm{C} 9$ & $0.066(6)$ & $0.031(4)$ & $0.040(5)$ & $0.005(4)$ & $0.001(5)$ & -0.005 \\
\hline $\mathrm{C} 10$ & $0.057(6)$ & $0.037(4)$ & $0.039(5)$ & 0.007 (4) & 0.009 (4) & 0.007 (4) \\
\hline $\mathrm{C} 11$ & $0.053(5)$ & $0.035(4)$ & $0.024(4)$ & $0.002(4)$ & $0.006(4)$ & -0.001 \\
\hline $\mathrm{C} 12$ & $0.047(5)$ & $0.027(4)$ & $0.015(4)$ & $0.007(3)$ & $-0.002(4)$ & $0.001(3)$ \\
\hline C13 & $0.047(5)$ & $0.029(3)$ & $0.025(4)$ & -0.003 & $0.003(4)$ & $-0.007(3)$ \\
\hline C14 & $0.038(5)$ & $0.029(3)$ & $0.025(5)$ & $0.005(3)$ & $0.006(4)$ & -0.005 \\
\hline $\mathrm{C} 15$ & $0.047(5)$ & $0.035(4)$ & $0.028(4)$ & $0.005(4)$ & $0.007(4)$ & -0.003 \\
\hline $\mathrm{C} 16$ & $0.063(6)$ & $0.040(4)$ & $0.042(5)$ & -0.008 & $0.000(5)$ & -0.004 \\
\hline $\mathrm{C} 17$ & $0.052(5)$ & $0.038(4)$ & $0.038(5)$ & $0.001(4)$ & $0.012(4)$ & $0.003(4)$ \\
\hline $\mathrm{C} 18$ & $0.041(5)$ & $0.040(4)$ & $0.036(5)$ & $-0.003(4)$ & $0.002(4)$ & $-0.006(3)$ \\
\hline $\mathrm{O} 1 \mathrm{~W}$ & $0.066(4)$ & $0.036(3)$ & $0.051(4)$ & $-0.006(3)$ & $0.013(3)$ & $-0.001(2)$ \\
\hline $\mathrm{O} 2 \mathrm{~W}$ & $0.061(4)$ & $0.036(3)$ & $0.047(3)$ & $0.000(2)$ & $0.007(3)$ & $0.001(2)$ \\
\hline
\end{tabular}


Geometric parameters $\left(\AA,{ }^{\circ}\right)$

\begin{tabular}{|c|c|c|c|}
\hline $\mathrm{O} 1-\mathrm{C} 5$ & $1.245(7)$ & $\mathrm{C} 6-\mathrm{H} 6 \mathrm{~B}$ & 0.9900 \\
\hline $\mathrm{O} 2-\mathrm{C} 12$ & $1.248(7)$ & $\mathrm{C} 7-\mathrm{C} 8$ & $1.370(9)$ \\
\hline $\mathrm{N} 1-\mathrm{C} 4$ & $1.331(8)$ & $\mathrm{C} 7-\mathrm{C} 11$ & $1.398(9)$ \\
\hline $\mathrm{N} 1-\mathrm{C} 1$ & $1.364(8)$ & $\mathrm{C} 8-\mathrm{C} 9$ & $1.364(10)$ \\
\hline $\mathrm{N} 2-\mathrm{C} 3$ & $1.346(9)$ & $\mathrm{C} 8-\mathrm{H} 8$ & 0.9500 \\
\hline $\mathrm{N} 2-\mathrm{C} 2$ & $1.351(8)$ & $\mathrm{C} 9-\mathrm{H} 9$ & 0.9500 \\
\hline $\mathrm{N} 3-\mathrm{C} 5$ & $1.347(8)$ & $\mathrm{C} 10-\mathrm{C} 11$ & $1.378(10)$ \\
\hline $\mathrm{N} 3-\mathrm{C} 6$ & $1.456(8)$ & $\mathrm{C} 10-\mathrm{H} 10$ & 0.9500 \\
\hline N3-H3N & $0.88(3)$ & $\mathrm{C} 11-\mathrm{H} 11$ & 0.9500 \\
\hline $\mathrm{N} 4-\mathrm{C} 10$ & $1.330(9)$ & $\mathrm{C} 13-\mathrm{C} 14$ & $1.491(8)$ \\
\hline $\mathrm{N} 4-\mathrm{C} 9$ & $1.354(10)$ & $\mathrm{C} 13-\mathrm{H} 13 \mathrm{~A}$ & 0.9900 \\
\hline $\mathrm{N} 5-\mathrm{C} 12$ & $1.325(8)$ & $\mathrm{C} 13-\mathrm{H} 13 \mathrm{~B}$ & 0.9900 \\
\hline N5-C13 & $1.458(8)$ & $\mathrm{C} 14-\mathrm{C} 15$ & $1.366(10)$ \\
\hline $\mathrm{N} 5-\mathrm{H} 5 \mathrm{~N}$ & $0.88(3)$ & $\mathrm{C} 14-\mathrm{C} 18$ & $1.389(10)$ \\
\hline N6-C17 & $1.340(10)$ & $\mathrm{C} 15-\mathrm{C} 16$ & $1.386(9)$ \\
\hline $\mathrm{N} 6-\mathrm{C} 16$ & $1.344(10)$ & $\mathrm{C} 15-\mathrm{H} 15$ & 0.9500 \\
\hline $\mathrm{C} 1-\mathrm{C} 2$ & $1.377(9)$ & $\mathrm{C} 16-\mathrm{H} 16$ & 0.9500 \\
\hline $\mathrm{C} 1-\mathrm{C} 5$ & $1.485(8)$ & $\mathrm{C} 17-\mathrm{C} 18$ & $1.397(10)$ \\
\hline $\mathrm{C} 2-\mathrm{C} 12$ & $1.518(9)$ & C17-H17 & 0.9500 \\
\hline $\mathrm{C} 3-\mathrm{C} 4$ & $1.375(10)$ & $\mathrm{C} 18-\mathrm{H} 18$ & 0.9500 \\
\hline $\mathrm{C} 3-\mathrm{H} 3$ & 0.9500 & $\mathrm{O} 1 \mathrm{~W}-\mathrm{H} 1 \mathrm{WA}$ & 0.8569 \\
\hline $\mathrm{C} 4-\mathrm{H} 4$ & 0.9500 & $\mathrm{O} 1 \mathrm{~W}-\mathrm{H} 1 \mathrm{WB}$ & 0.9371 \\
\hline $\mathrm{C} 6-\mathrm{C} 7$ & $1.510(9)$ & $\mathrm{O} 2 \mathrm{~W}-\mathrm{H} 2 \mathrm{WA}$ & 0.8649 \\
\hline C6-H6A & 0.9900 & $\mathrm{O} 2 \mathrm{~W}-\mathrm{H} 2 \mathrm{WB}$ & 0.8483 \\
\hline $\mathrm{C} 4-\mathrm{N} 1-\mathrm{C} 1$ & $115.9(6)$ & $\mathrm{C} 7-\mathrm{C} 8-\mathrm{H} 8$ & 119.9 \\
\hline $\mathrm{C} 3-\mathrm{N} 2-\mathrm{C} 2$ & $114.8(6)$ & $\mathrm{N} 4-\mathrm{C} 9-\mathrm{C} 8$ & $123.9(7)$ \\
\hline $\mathrm{C} 5-\mathrm{N} 3-\mathrm{C} 6$ & $122.5(5)$ & $\mathrm{N} 4-\mathrm{C} 9-\mathrm{H} 9$ & 118.1 \\
\hline $\mathrm{C} 5-\mathrm{N} 3-\mathrm{H} 3 \mathrm{~N}$ & $99(4)$ & $\mathrm{C} 8-\mathrm{C} 9-\mathrm{H} 9$ & 118.1 \\
\hline $\mathrm{C} 6-\mathrm{N} 3-\mathrm{H} 3 \mathrm{~N}$ & $139(5)$ & $\mathrm{N} 4-\mathrm{C} 10-\mathrm{C} 11$ & $125.2(7)$ \\
\hline $\mathrm{C} 10-\mathrm{N} 4-\mathrm{C} 9$ & $115.0(6)$ & $\mathrm{N} 4-\mathrm{C} 10-\mathrm{H} 10$ & 117.4 \\
\hline $\mathrm{C} 12-\mathrm{N} 5-\mathrm{C} 13$ & $122.6(6)$ & $\mathrm{C} 11-\mathrm{C} 10-\mathrm{H} 10$ & 117.4 \\
\hline $\mathrm{C} 12-\mathrm{N} 5-\mathrm{H} 5 \mathrm{~N}$ & $128(4)$ & $\mathrm{C} 10-\mathrm{C} 11-\mathrm{C} 7$ & $118.3(6)$ \\
\hline $\mathrm{C} 13-\mathrm{N} 5-\mathrm{H} 5 \mathrm{~N}$ & $106(4)$ & $\mathrm{C} 10-\mathrm{C} 11-\mathrm{H} 11$ & 120.8 \\
\hline $\mathrm{C} 17-\mathrm{N} 6-\mathrm{C} 16$ & $116.6(6)$ & $\mathrm{C} 7-\mathrm{C} 11-\mathrm{H} 11$ & 120.8 \\
\hline $\mathrm{N} 1-\mathrm{C} 1-\mathrm{C} 2$ & $120.9(6)$ & $\mathrm{O} 2-\mathrm{C} 12-\mathrm{N} 5$ & $123.9(6)$ \\
\hline $\mathrm{N} 1-\mathrm{C} 1-\mathrm{C} 5$ & $116.8(6)$ & $\mathrm{O} 2-\mathrm{C} 12-\mathrm{C} 2$ & $119.7(6)$ \\
\hline $\mathrm{C} 2-\mathrm{C} 1-\mathrm{C} 5$ & $122.2(5)$ & $\mathrm{N} 5-\mathrm{C} 12-\mathrm{C} 2$ & $116.2(6)$ \\
\hline $\mathrm{N} 2-\mathrm{C} 2-\mathrm{C} 1$ & $123.1(5)$ & $\mathrm{N} 5-\mathrm{C} 13-\mathrm{C} 14$ & $112.5(6)$ \\
\hline $\mathrm{N} 2-\mathrm{C} 2-\mathrm{C} 12$ & $111.4(6)$ & $\mathrm{N} 5-\mathrm{C} 13-\mathrm{H} 13 \mathrm{~A}$ & 109.1 \\
\hline $\mathrm{C} 1-\mathrm{C} 2-\mathrm{C} 12$ & $125.5(6)$ & $\mathrm{C} 14-\mathrm{C} 13-\mathrm{H} 13 \mathrm{~A}$ & 109.1 \\
\hline $\mathrm{N} 2-\mathrm{C} 3-\mathrm{C} 4$ & $122.5(7)$ & N5 $-\mathrm{C} 13-\mathrm{H} 13 \mathrm{~B}$ & 109.1 \\
\hline $\mathrm{N} 2-\mathrm{C} 3-\mathrm{H} 3$ & 118.7 & $\mathrm{C} 14-\mathrm{C} 13-\mathrm{H} 13 \mathrm{~B}$ & 109.1 \\
\hline $\mathrm{C} 4-\mathrm{C} 3-\mathrm{H} 3$ & 118.7 & $\mathrm{H} 13 \mathrm{~A}-\mathrm{C} 13-\mathrm{H} 13 \mathrm{~B}$ & 107.8 \\
\hline $\mathrm{N} 1-\mathrm{C} 4-\mathrm{C} 3$ & $122.6(6)$ & $\mathrm{C} 15-\mathrm{C} 14-\mathrm{C} 18$ & $116.6(6)$ \\
\hline $\mathrm{N} 1-\mathrm{C} 4-\mathrm{H} 4$ & 118.7 & $\mathrm{C} 15-\mathrm{C} 14-\mathrm{C} 13$ & $121.9(6)$ \\
\hline
\end{tabular}




\begin{tabular}{|c|c|}
\hline $\mathrm{C} 3-\mathrm{C} 4-\mathrm{H} 4$ & 118.7 \\
\hline $\mathrm{O} 1-\mathrm{C} 5-\mathrm{N} 3$ & $123.0(6)$ \\
\hline $\mathrm{O} 1-\mathrm{C} 5-\mathrm{C} 1$ & $120.7(6)$ \\
\hline $\mathrm{N} 3-\mathrm{C} 5-\mathrm{C} 1$ & $116.3(5)$ \\
\hline $\mathrm{N} 3-\mathrm{C} 6-\mathrm{C} 7$ & $112.7(6)$ \\
\hline $\mathrm{N} 3-\mathrm{C} 6-\mathrm{H} 6 \mathrm{~A}$ & 109.1 \\
\hline $\mathrm{C} 7-\mathrm{C} 6-\mathrm{H} 6 \mathrm{~A}$ & 109.1 \\
\hline $\mathrm{N} 3-\mathrm{C} 6-\mathrm{H} 6 \mathrm{~B}$ & 109.1 \\
\hline $\mathrm{C} 7-\mathrm{C} 6-\mathrm{H} 6 \mathrm{~B}$ & 109.1 \\
\hline $\mathrm{H} 6 \mathrm{~A}-\mathrm{C} 6-\mathrm{H} 6 \mathrm{~B}$ & 107.8 \\
\hline $\mathrm{C} 8-\mathrm{C} 7-\mathrm{C} 11$ & $117.3(6)$ \\
\hline $\mathrm{C} 8-\mathrm{C} 7-\mathrm{C} 6$ & $121.6(6)$ \\
\hline $\mathrm{C} 11-\mathrm{C} 7-\mathrm{C} 6$ & $121.1(6)$ \\
\hline $\mathrm{C} 9-\mathrm{C} 8-\mathrm{C} 7$ & $120.3(7)$ \\
\hline $\mathrm{C} 9-\mathrm{C} 8-\mathrm{H} 8$ & 119.9 \\
\hline $\mathrm{C} 4-\mathrm{N} 1-\mathrm{C} 1-\mathrm{C} 2$ & $0.2(9)$ \\
\hline $\mathrm{C} 4-\mathrm{N} 1-\mathrm{C} 1-\mathrm{C} 5$ & $178.1(6)$ \\
\hline $\mathrm{C} 3-\mathrm{N} 2-\mathrm{C} 2-\mathrm{C} 1$ & $0.9(10)$ \\
\hline $\mathrm{C} 3-\mathrm{N} 2-\mathrm{C} 2-\mathrm{C} 12$ & $179.9(6)$ \\
\hline $\mathrm{N} 1-\mathrm{C} 1-\mathrm{C} 2-\mathrm{N} 2$ & $0.3(10)$ \\
\hline $\mathrm{C} 5-\mathrm{C} 1-\mathrm{C} 2-\mathrm{N} 2$ & $-177.6(6)$ \\
\hline $\mathrm{N} 1-\mathrm{C} 1-\mathrm{C} 2-\mathrm{C} 12$ & $-178.5(6)$ \\
\hline $\mathrm{C} 5-\mathrm{C} 1-\mathrm{C} 2-\mathrm{C} 12$ & $3.6(10)$ \\
\hline $\mathrm{C} 2-\mathrm{N} 2-\mathrm{C} 3-\mathrm{C} 4$ & $-2.5(11)$ \\
\hline $\mathrm{C} 1-\mathrm{N} 1-\mathrm{C} 4-\mathrm{C} 3$ & $-1.8(10)$ \\
\hline $\mathrm{N} 2-\mathrm{C} 3-\mathrm{C} 4-\mathrm{N} 1$ & $3.2(12)$ \\
\hline $\mathrm{C} 6-\mathrm{N} 3-\mathrm{C} 5-\mathrm{O} 1$ & $2.1(10)$ \\
\hline $\mathrm{C} 6-\mathrm{N} 3-\mathrm{C} 5-\mathrm{C} 1$ & $-175.4(5)$ \\
\hline $\mathrm{N} 1-\mathrm{C} 1-\mathrm{C} 5-\mathrm{O} 1$ & $-161.3(6)$ \\
\hline $\mathrm{C} 2-\mathrm{C} 1-\mathrm{C} 5-\mathrm{O} 1$ & $16.6(9)$ \\
\hline $\mathrm{N} 1-\mathrm{C} 1-\mathrm{C} 5-\mathrm{N} 3$ & $16.2(8)$ \\
\hline $\mathrm{C} 2-\mathrm{C} 1-\mathrm{C} 5-\mathrm{N} 3$ & $-165.8(6)$ \\
\hline $\mathrm{C} 5-\mathrm{N} 3-\mathrm{C} 6-\mathrm{C} 7$ & $-79.3(7)$ \\
\hline $\mathrm{N} 3-\mathrm{C} 6-\mathrm{C} 7-\mathrm{C} 8$ & $94.4(7)$ \\
\hline $\mathrm{N} 3-\mathrm{C} 6-\mathrm{C} 7-\mathrm{C} 11$ & $-87.3(8)$ \\
\hline $\mathrm{C} 11-\mathrm{C} 7-\mathrm{C} 8-\mathrm{C} 9$ & $0.1(11)$ \\
\hline $\mathrm{C} 6-\mathrm{C} 7-\mathrm{C} 8-\mathrm{C} 9$ & $178.5(7)$ \\
\hline $\mathrm{C} 10-\mathrm{N} 4-\mathrm{C} 9-\mathrm{C} 8$ & $-3.8(11)$ \\
\hline
\end{tabular}

C18-C14-C13

C14-C15-C16

C14-C15-H15

$\mathrm{C} 16-\mathrm{C} 15-\mathrm{H} 15$

$\mathrm{N} 6-\mathrm{C} 16-\mathrm{C} 15$

N6-C16-H16

$\mathrm{C} 15-\mathrm{C} 16-\mathrm{H} 16$

N6- C17-C18

N6- $17-\mathrm{H} 17$

C18-C17-H17

C14-C18-C17

C14-C18-H18

C17-C18-H18

H1WA-O1W-H1WB

$\mathrm{H} 2 \mathrm{WA}-\mathrm{O} 2 \mathrm{~W}-\mathrm{H} 2 \mathrm{WB}$

$\mathrm{C} 7-\mathrm{C} 8-\mathrm{C} 9-\mathrm{N} 4$

C9-N4- $10-\mathrm{C} 11$

$\mathrm{N} 4-\mathrm{C} 10-\mathrm{C} 11-\mathrm{C} 7$

$\mathrm{C} 8-\mathrm{C} 7-\mathrm{C} 11-\mathrm{C} 10$

C6-C7- $11-\mathrm{C} 10$

$\mathrm{C} 13-\mathrm{N} 5-\mathrm{C} 12-\mathrm{O} 2$

$\mathrm{C} 13-\mathrm{N} 5-\mathrm{C} 12-\mathrm{C} 2$

$\mathrm{N} 2-\mathrm{C} 2-\mathrm{C} 12-\mathrm{O} 2$

$\mathrm{C} 1-\mathrm{C} 2-\mathrm{C} 12-\mathrm{O} 2$

$\mathrm{N} 2-\mathrm{C} 2-\mathrm{C} 12-\mathrm{N} 5$

$\mathrm{C} 1-\mathrm{C} 2-\mathrm{C} 12-\mathrm{N} 5$

$\mathrm{C} 12-\mathrm{N} 5-\mathrm{C} 13-\mathrm{C} 14$

N5-C13-C14-C15

N5-C13-C14-C18

C18-C14-C15-C16

$\mathrm{C} 13-\mathrm{C} 14-\mathrm{C} 15-\mathrm{C} 16$

C17-N6-C16-C15

$\mathrm{C} 14-\mathrm{C} 15-\mathrm{C} 16-\mathrm{N} 6$

C16-N6-C17- 18

$\mathrm{C} 15-\mathrm{C} 14-\mathrm{C} 18-\mathrm{C} 17$

$\mathrm{C} 13-\mathrm{C} 14-\mathrm{C} 18-\mathrm{C} 17$

N6-C17-C18-C14
$121.5(6)$

$121.0(7)$

119.5

119.5

$122.9(7)$

118.6

118.6

$123.0(8)$

118.5

118.5

$119.9(7)$

120.1

120.1

113.1

105.0

$2.1(12)$

$3.4(12)$

$-1.4(12)$

$-0.5(11)$

$-178.9(6)$

$-4.5(10)$

171.7 (6)

$78.2(8)$

$-102.8(8)$

$-98.1(7)$

$80.8(9)$

$148.9(6)$

$141.5(7)$

$-40.3(9)$

$1.4(10)$

$179.6(6)$

$-0.6(11)$

$-0.9(12)$

$1.6(11)$

$-0.4(10)$

$-178.7(6)$

$-1.2(11)$

Hydrogen-bond geometry $\left(A,{ }^{\circ}\right)$

\begin{tabular}{lllll}
\hline$D-\mathrm{H} \cdots A$ & $D-\mathrm{H}$ & $\mathrm{H} \cdots A$ & $D \cdots A$ & $D-\mathrm{H}^{\cdots} \cdot A$ \\
\hline $\mathrm{N} 3-\mathrm{H} 3 N \cdots \mathrm{N} 1$ & $0.88(3)$ & $2.08(5)$ & $2.718(7)$ & $129(5)$ \\
$\mathrm{N} 5-\mathrm{H} 5 N \cdots \mathrm{O} 1^{\mathrm{i}}$ & $0.88(3)$ & $2.05(4)$ & $2.858(7)$ & $151(6)$ \\
$\mathrm{O} 1 W-\mathrm{H} 1 W A \cdots \mathrm{O} 2 W^{\mathrm{ii}}$ & 0.86 & 1.91 & $2.762(7)$ & 177 \\
$\mathrm{O} 1 W-\mathrm{H} 1 W B \cdots \mathrm{N} 6^{\text {iii }}$ & 0.94 & 1.97 & $2.886(7)$ & 164 \\
$\mathrm{O} 2 W-\mathrm{H} 2 W A \cdots \mathrm{O} 1 W^{\text {iv }}$ & 0.86 & 1.91 & $2.765(8)$ & 172
\end{tabular}




$\begin{array}{lllll}\mathrm{O} 2 W-\mathrm{H} 2 W B \cdots \mathrm{N} 4 & 0.85 & 2.06 & 2.888(7) & 164 \\ \mathrm{C} 3-\mathrm{H} 3 \cdots \mathrm{O} 1 W^{\mathrm{v}} & 0.95 & 2.38 & 3.273(8) & 156 \\ \mathrm{C} 4-\mathrm{H} 4 \cdots \mathrm{O} 2 W^{\mathrm{ii}} & 0.95 & 2.58 & 3.253(8) & 128 \\ \mathrm{C} 6-\mathrm{H} 6 A \cdots \mathrm{N} 2^{\text {vii }} & 0.99 & 2.57 & 3.515(8) & 159 \\ \mathrm{C} 16-\mathrm{H} 16 \cdots \mathrm{N} 4^{\mathrm{iv}} & 0.95 & 2.60 & 3.451(9) & 149\end{array}$

Symmetry codes: (i) $x-1, y, z$; (ii) $x,-y+1, z+1 / 2$; (iii) $x+1,-y+1, z+1 / 2$; (iv) $x-1,-y+1, z-1 / 2$; (v) $x-1,-y+2, z-1 / 2$; (vi) $x, y+1, z$; (vii) $x+1,-y+2, z+1 / 2$.

(I) Poly[[[ $\mu_{4}-N^{2}, N^{3}$-bis(pyridin-4-ylmethyl) pyrazine-2,3-dicarboxamide]silver(I)] nitrate]

Crystal data

$\left[\mathrm{Ag}\left(\mathrm{C}_{18} \mathrm{H}_{16} \mathrm{~N}_{6} \mathrm{O}_{2}\right)\right] \mathrm{NO}_{3}$

$M_{r}=518.25$

Orthorhombic, Fddd

$a=14.9776(16) \AA$

$b=17.3228(12) \AA$

$c=29.570(4) \AA$

$V=7672.1(14) \AA^{3}$

$Z=16$

$F(000)=4160$

Data collection

Stoe IPDS 1

diffractometer

Radiation source: fine-focus sealed tube

Plane graphite monochromator

$\varphi$ rotation scans

Absorption correction: multi-scan

(MULABS; Spek, 2009)

$T_{\min }=0.985, T_{\max }=1.000$

Refinement

Refinement on $F^{2}$

Least-squares matrix: full

$R\left[F^{2}>2 \sigma\left(F^{2}\right)\right]=0.045$

$w R\left(F^{2}\right)=0.107$

$S=0.90$

1721 reflections

141 parameters

0 restraints

Primary atom site location: structure-invariant direct methods
$D_{\mathrm{x}}=1.795 \mathrm{Mg} \mathrm{m}^{-3}$

Mo $K \alpha$ radiation, $\lambda=0.71073 \AA$

Cell parameters from 6115 reflections

$\theta=1.9-25.9^{\circ}$

$\mu=1.10 \mathrm{~mm}^{-1}$

$T=153 \mathrm{~K}$

Block, colourless

$0.40 \times 0.30 \times 0.20 \mathrm{~mm}$

13548 measured reflections

1721 independent reflections

1038 reflections with $I>2 \sigma(I)$

$R_{\text {int }}=0.096$

$\theta_{\max }=25.3^{\circ}, \theta_{\min }=2.7^{\circ}$

$h=-17 \rightarrow 17$

$k=-20 \rightarrow 20$

$l=-35 \rightarrow 35$

Secondary atom site location: difference Fourier map

Hydrogen site location: inferred from neighbouring sites

$\mathrm{H}$-atom parameters constrained

$w=1 /\left[\sigma^{2}\left(F_{\mathrm{o}}{ }^{2}\right)+(0.0575 P)^{2}\right]$

where $P=\left(F_{\mathrm{o}}^{2}+2 F_{\mathrm{c}}^{2}\right) / 3$

$(\Delta / \sigma)_{\max }=0.003$

$\Delta \rho_{\max }=0.68$ e $\AA^{-3}$

$\Delta \rho_{\min }=-0.65$ e $\AA^{-3}$

\section{Special details}

Geometry. Bond distances, angles etc. have been calculated using the rounded fractional coordinates. All su's are estimated from the variances of the (full) variance-covariance matrix. The cell esds are taken into account in the estimation of distances, angles and torsion angles

Fractional atomic coordinates and isotropic or equivalent isotropic displacement parameters $\left(\AA^{2}\right)$

\begin{tabular}{llllll}
\hline & $x$ & $y$ & $z$ & $U_{\text {iso }} * U_{\text {eq }}$ & Occ. $(<1)$ \\
\hline Ag1 & 0.87500 & $0.96632(4)$ & 0.37500 & $0.0464(2)$ & \\
O1 & $0.9796(3)$ & $0.8101(3)$ & $0.14979(13)$ & $0.0483(14)$ & \\
N1 & $0.9673(3)$ & $0.8836(3)$ & $0.04722(14)$ & $0.0447(18)$ &
\end{tabular}




\begin{tabular}{|c|c|c|c|c|c|}
\hline N3 & $1.0135(3)$ & $0.9361(3)$ & $0.14261(16)$ & $0.050(2)$ & \\
\hline N4 & 0.9359 & $0.9589(3)$ & $0.31086(16)$ & $0.0427(17)$ & \\
\hline $\mathrm{C} 1$ & $0.9207(3)$ & $0.8777(4)$ & $0.08584(15)$ & $0.0337(16)$ & \\
\hline $\mathrm{C} 4$ & $0.9204(4)$ & 0.8805 (4) & 0.00937 (17) & 0.0453 (19) & \\
\hline C5 & $0.9745(3)$ & 0.8715 (4) & $0.12935(16)$ & $0.0340(18)$ & \\
\hline C6 & $1.0689(4)$ & $0.9393(4)$ & $0.18277(18)$ & $0.051(2)$ & \\
\hline $\mathrm{C} 7$ & $1.0197(4)$ & $0.9446(3)$ & $0.22692(19)$ & 0.0437 (19) & \\
\hline $\mathrm{C} 8$ & $1.0640(4)$ & $0.9276(4)$ & $0.26675(19)$ & $0.045(2)$ & \\
\hline C9 & $1.0221(4)$ & $0.9354(4)$ & $0.3075(2)$ & $0.046(2)$ & \\
\hline $\mathrm{C} 10$ & $0.8921(3)$ & 0.9745 & $0.27201(19)$ & $0.043(2)$ & \\
\hline $\mathrm{C} 11$ & $0.9302(4)$ & $0.9686(4)$ & $0.23033(19)$ & 0.0447 (19) & \\
\hline $\mathrm{O} 11$ & $0.9918(8)$ & $0.0666(8)$ & $0.0912(4)$ & $0.089(3)$ & 0.500 \\
\hline $\mathrm{O} 12$ & $1.0655(16)$ & $0.1323(15)$ & $0.1063(6)$ & 0.089 (3) & 0.250 \\
\hline $\mathrm{O} 13$ & $0.9326(9)$ & $0.0862(7)$ & $0.1489(4)$ & 0.089 (3) & 0.500 \\
\hline O14 & $0.9869(18)$ & 0.1233 (19) & $0.1570(7)$ & $0.089(3)$ & 0.250 \\
\hline N10A & $0.9614(15)$ & 0.12500 & 0.12500 & 0.089 (3) & 0.500 \\
\hline N10B & $0.9960(16)$ & 0.12500 & 0.12500 & $0.089(3)$ & 0.500 \\
\hline $\mathrm{H} 4$ & 0.95046 & 0.88600 & -0.01873 & $0.0540^{*}$ & \\
\hline $\mathrm{H} 3 \mathrm{~N}$ & 1.00563 & 0.97838 & 0.12661 & $0.0600 *$ & \\
\hline H6A & 1.10904 & 0.98449 & 0.18023 & $0.0610 *$ & \\
\hline H6B & 1.10697 & 0.89253 & 0.18345 & $0.0610 *$ & \\
\hline H8 & 1.12416 & 0.91024 & 0.26574 & $0.0540 *$ & \\
\hline H9 & 1.05428 & 0.92399 & 0.33436 & $0.0560^{*}$ & \\
\hline $\mathrm{H} 10$ & 0.83154 & 0.99047 & 0.27385 & $0.0520 *$ & \\
\hline H11 & 0.89687 & 0.98062 & 0.20392 & $0.0530 *$ & \\
\hline
\end{tabular}

Atomic displacement parameters $\left(\AA^{2}\right)$

\begin{tabular}{lllllll}
\hline & $U^{11}$ & $U^{22}$ & $U^{33}$ & $U^{12}$ & $U^{13}$ & $U^{23}$ \\
\hline Ag1 & $0.0474(4)$ & $0.0397(4)$ & $0.0521(4)$ & 0.0000 & $0.0035(4)$ & 0.0000 \\
O1 & $0.048(2)$ & $0.054(3)$ & $0.043(2)$ & $0.000(2)$ & $-0.005(2)$ & $0.010(2)$ \\
N1 & $0.047(3)$ & $0.057(4)$ & $0.030(2)$ & $0.003(3)$ & $0.004(2)$ & $-0.004(2)$ \\
N3 & $0.078(4)$ & $0.035(4)$ & $0.037(3)$ & $0.002(3)$ & $-0.015(3)$ & $-0.006(2)$ \\
N4 & $0.042(3)$ & $0.039(3)$ & $0.047(3)$ & $0.001(2)$ & $-0.009(2)$ & $-0.006(2)$ \\
C1 & $0.037(2)$ & $0.035(3)$ & $0.029(3)$ & $0.005(3)$ & $0.002(2)$ & $-0.010(3)$ \\
C4 & $0.059(3)$ & $0.047(4)$ & $0.030(3)$ & $0.009(4)$ & $0.006(2)$ & $0.000(3)$ \\
C5 & $0.030(2)$ & $0.046(4)$ & $0.026(3)$ & $0.005(2)$ & $0.010(2)$ & $-0.007(4)$ \\
C6 & $0.062(4)$ & $0.057(5)$ & $0.033(3)$ & $-0.003(3)$ & $-0.008(3)$ & $-0.006(3)$ \\
C7 & $0.051(3)$ & $0.036(4)$ & $0.044(3)$ & $-0.004(3)$ & $-0.012(3)$ & $-0.008(3)$ \\
C8 & $0.046(3)$ & $0.044(4)$ & $0.045(4)$ & $0.007(3)$ & $-0.006(3)$ & $-0.004(3)$ \\
C9 & $0.051(4)$ & $0.051(4)$ & $0.037(3)$ & $0.009(3)$ & $-0.008(3)$ & $0.000(3)$ \\
C10 & $0.037(4)$ & $0.041(4)$ & $0.052(3)$ & $-0.004(3)$ & $-0.010(3)$ & $-0.006(3)$ \\
C11 & $0.050(3)$ & $0.045(4)$ & $0.039(3)$ & $-0.001(3)$ & $-0.015(3)$ & $-0.008(3)$ \\
O11 & $0.112(6)$ & $0.088(6)$ & $0.067(4)$ & 0.0000 & 0.0000 & $0.028(4)$ \\
O12 & $0.112(6)$ & $0.088(6)$ & $0.067(4)$ & 0.0000 & 0.0000 & $0.028(4)$ \\
O13 & $0.112(6)$ & $0.088(6)$ & $0.067(4)$ & 0.0000 & 0.0000 & $0.028(4)$ \\
O14 & $0.112(6)$ & $0.088(6)$ & $0.067(4)$ & 0.0000 & 0.0000 & $0.028(4)$ \\
N10A & $0.112(6)$ & $0.088(6)$ & $0.067(4)$ & 0.0000 & 0.0000 & $0.028(4)$
\end{tabular}


Geometric parameters $\left(\AA,{ }^{\circ}\right)$

\begin{tabular}{|c|c|c|c|}
\hline $\mathrm{Ag} 1-\mathrm{Ag} 1^{\mathrm{i}}$ & $3.1638(11)$ & $\mathrm{C} 8-\mathrm{C} 9$ & $1.365(8)$ \\
\hline $\mathrm{Ag} 1-\mathrm{N} 4$ & $2.109(5)$ & $\mathrm{C} 10-\mathrm{C} 11$ & $1.362(8)$ \\
\hline $\mathrm{Ag} 1-\mathrm{O} 1^{\mathrm{ii}}$ & $2.814(5)$ & $\mathrm{O} 11-\mathrm{N} 10 \mathrm{~A}$ & $1.493(14)$ \\
\hline $\mathrm{Ag} 1-\mathrm{O} 1^{\mathrm{iii}}$ & $2.814(5)$ & $\mathrm{O} 11-\mathrm{N} 10 \mathrm{~B}$ & $1.424(13)$ \\
\hline $\mathrm{Ag} 1-\mathrm{N} 4^{\mathrm{iv}}$ & $2.109(5)$ & $\mathrm{O} 11-\mathrm{O} 12$ & $1.65(3)$ \\
\hline $\mathrm{O} 1-\mathrm{C} 5$ & $1.226(8)$ & $\mathrm{O} 12-\mathrm{N} 10 \mathrm{~B}$ & $1.19(3)$ \\
\hline $\mathrm{N} 1-\mathrm{C} 1$ & $1.342(6)$ & $\mathrm{O} 12-\mathrm{N} 10 \mathrm{~A}$ & $1.66(3)$ \\
\hline $\mathrm{N} 1-\mathrm{C} 4$ & $1.323(7)$ & $\mathrm{O} 13-\mathrm{N} 10 \mathrm{~A}$ & $1.066(15)$ \\
\hline $\mathrm{N} 3-\mathrm{C} 5$ & $1.322(8)$ & O13-N10B & $1.36(2)$ \\
\hline $\mathrm{N} 3-\mathrm{C} 6$ & $1.450(7)$ & $\mathrm{O} 13-\mathrm{O} 14$ & $1.06(3)$ \\
\hline $\mathrm{N} 4-\mathrm{C} 9$ & $1.357(8)$ & $\mathrm{O} 14-\mathrm{N} 10 \mathrm{~B}$ & $0.96(2)$ \\
\hline $\mathrm{N} 4-\mathrm{C} 10$ & $1.350(7)$ & $\mathrm{O} 14-\mathrm{N} 10 \mathrm{~A}$ & $1.02(2)$ \\
\hline $\mathrm{C} 1-\mathrm{C} 5$ & $1.522(6)$ & $\mathrm{C} 4-\mathrm{H} 4$ & 0.9500 \\
\hline $\mathrm{C} 1-\mathrm{C} 1^{\mathrm{v}}$ & $1.372(6)$ & $\mathrm{C} 6-\mathrm{H} 6 \mathrm{~B}$ & 0.9900 \\
\hline $\mathrm{C} 4-\mathrm{C}^{\mathrm{v}}$ & $1.373(9)$ & $\mathrm{C} 6-\mathrm{H} 6 \mathrm{~A}$ & 0.9900 \\
\hline $\mathrm{N} 3-\mathrm{H} 3 \mathrm{~N}$ & 0.8800 & $\mathrm{C} 8-\mathrm{H} 8$ & 0.9500 \\
\hline $\mathrm{C} 6-\mathrm{C} 7$ & $1.502(8)$ & $\mathrm{C} 9-\mathrm{H} 9$ & 0.9500 \\
\hline $\mathrm{C} 7-\mathrm{C} 8$ & $1.384(8)$ & $\mathrm{C} 10-\mathrm{H} 10$ & 0.9500 \\
\hline $\mathrm{C} 7-\mathrm{C} 11$ & $1.407(8)$ & $\mathrm{C} 11-\mathrm{H} 11$ & 0.9500 \\
\hline $\mathrm{N} 4-\mathrm{Ag} 1-\mathrm{N} 4^{\mathrm{iv}}$ & $173.0(2)$ & $\mathrm{C} 6-\mathrm{C} 7-\mathrm{C} 8$ & $119.5(5)$ \\
\hline 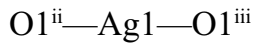 & $109.48(14)$ & $\mathrm{C} 6-\mathrm{C} 7-\mathrm{C} 11$ & $123.2(5)$ \\
\hline $\mathrm{Ag} 1-\mathrm{Ag} 1-\mathrm{N} 4$ & $86.51(14)$ & $\mathrm{C} 7-\mathrm{C} 8-\mathrm{C} 9$ & $120.7(6)$ \\
\hline $\mathrm{O} 1 \mathrm{ii}-\mathrm{Ag} 1-\mathrm{N} 4$ & $97.80(15)$ & $\mathrm{N} 4-\mathrm{C} 9-\mathrm{C} 8$ & $122.1(5)$ \\
\hline $\mathrm{O} 1{ }^{\mathrm{iii}}-\mathrm{Ag} 1-\mathrm{N} 4$ & $86.26(15)$ & $\mathrm{N} 4-\mathrm{C} 10-\mathrm{C} 11$ & $123.5(5)$ \\
\hline $\mathrm{Ag} 1^{\mathrm{i}}-\mathrm{Ag} 1-\mathrm{O} 1^{\mathrm{ii}}$ & $125.26(10)$ & $\mathrm{C} 7-\mathrm{C} 11-\mathrm{C} 10$ & $119.1(5)$ \\
\hline $\mathrm{Ag} 1^{\mathrm{i}}-\mathrm{Ag} 1-\mathrm{O} 1^{\mathrm{iii}}$ & $125.26(10)$ & $\mathrm{C} 4 \mathrm{v}-\mathrm{C} 4-\mathrm{H} 4$ & 119.00 \\
\hline $\operatorname{Ag} 1^{\mathrm{i}}-\mathrm{Ag} 1-\mathrm{N} 4^{\mathrm{iv}}$ & $86.51(14)$ & $\mathrm{N} 1-\mathrm{C} 4-\mathrm{H} 4$ & 119.00 \\
\hline $\mathrm{O} 1^{\mathrm{ii}}-\mathrm{Ag} 1-\mathrm{N} 4^{\mathrm{iv}}$ & $86.26(15)$ & $\mathrm{N} 3-\mathrm{C} 6-\mathrm{H} 6 \mathrm{~B}$ & 108.00 \\
\hline $\mathrm{O} 1^{\mathrm{iii}}-\mathrm{Ag} 1-\mathrm{N} 4^{\mathrm{iv}}$ & $97.80(15)$ & $\mathrm{C} 7-\mathrm{C} 6-\mathrm{H} 6 \mathrm{~A}$ & 108.00 \\
\hline $\mathrm{Ag} 1^{\mathrm{vi}}-\mathrm{O} 1-\mathrm{C} 5$ & $114.9(3)$ & $\mathrm{H} 6 \mathrm{~A}-\mathrm{C} 6-\mathrm{H} 6 \mathrm{~B}$ & 107.00 \\
\hline $\mathrm{C} 1-\mathrm{N} 1-\mathrm{C} 4$ & $116.2(5)$ & $\mathrm{C} 7-\mathrm{C} 6-\mathrm{H} 6 \mathrm{~B}$ & 108.00 \\
\hline $\mathrm{C} 5-\mathrm{N} 3-\mathrm{C} 6$ & $121.9(5)$ & $\mathrm{N} 3-\mathrm{C} 6-\mathrm{H} 6 \mathrm{~A}$ & 108.00 \\
\hline $\mathrm{Ag} 1-\mathrm{N} 4-\mathrm{C} 9$ & $119.7(4)$ & $\mathrm{C} 7-\mathrm{C} 8-\mathrm{H} 8$ & 120.00 \\
\hline $\mathrm{Ag} 1-\mathrm{N} 4-\mathrm{C} 10$ & $122.9(3)$ & $\mathrm{C} 9-\mathrm{C} 8-\mathrm{H} 8$ & 120.00 \\
\hline $\mathrm{C} 9-\mathrm{N} 4-\mathrm{C} 10$ & $117.4(5)$ & $\mathrm{C} 8-\mathrm{C} 9-\mathrm{H} 9$ & 119.00 \\
\hline $\mathrm{N} 1-\mathrm{C} 1-\mathrm{C} 5$ & $116.7(4)$ & $\mathrm{N} 4-\mathrm{C} 9-\mathrm{H} 9$ & 119.00 \\
\hline $\mathrm{N} 1-\mathrm{C} 1-\mathrm{C}^{\mathrm{v}}$ & $121.6(4)$ & $\mathrm{N} 4-\mathrm{C} 10-\mathrm{H} 10$ & 118.00 \\
\hline $\mathrm{C} 1 \mathrm{v}-\mathrm{C} 1-\mathrm{C} 5$ & $121.6(4)$ & $\mathrm{C} 11-\mathrm{C} 10-\mathrm{H} 10$ & 118.00 \\
\hline $\mathrm{N} 1-\mathrm{C} 4-\mathrm{C}^{\mathrm{v}}$ & $122.1(5)$ & $\mathrm{O} 11-\mathrm{N} 10 \mathrm{~A}-\mathrm{O} 13$ & $98.0(8)$ \\
\hline $\mathrm{C} 5-\mathrm{N} 3-\mathrm{H} 3 \mathrm{~N}$ & 119.00 & $\mathrm{O} 13-\mathrm{N} 10 \mathrm{~A}-\mathrm{N} 10 \mathrm{~B}$ & $113.9(13)$ \\
\hline $\mathrm{C} 6-\mathrm{N} 3-\mathrm{H} 3 \mathrm{~N}$ & 119.00 & $\mathrm{O} 11-\mathrm{N} 10 \mathrm{~A}-\mathrm{N} 10 \mathrm{~B}$ & $72.3(9)$ \\
\hline $\mathrm{O} 1-\mathrm{C} 5-\mathrm{N} 3$ & $124.1(5)$ & $\mathrm{O} 11-\mathrm{N} 10 \mathrm{~B}-\mathrm{N} 10 \mathrm{~A}$ & $87.5(11)$ \\
\hline $\mathrm{O} 1-\mathrm{C} 5-\mathrm{C} 1$ & $120.7(6)$ & $\mathrm{O} 11-\mathrm{N} 10 \mathrm{~B}-\mathrm{O} 13$ & $89.0(10)$ \\
\hline
\end{tabular}




\begin{tabular}{|c|c|c|c|}
\hline $\mathrm{N} 3-\mathrm{C} 5-\mathrm{C} 1$ & $115.2(5)$ & $\mathrm{O} 13-\mathrm{N} 10 \mathrm{~B}-\mathrm{N} 10 \mathrm{~A}$ & $45.8(9)$ \\
\hline $\mathrm{N} 3-\mathrm{C} 6-\mathrm{C} 7$ & $115.7(5)$ & $\mathrm{C} 7-\mathrm{C} 11-\mathrm{H} 11$ & 120.00 \\
\hline $\mathrm{C} 8-\mathrm{C} 7-\mathrm{C} 11$ & $117.3(5)$ & $\mathrm{C} 10-\mathrm{C} 11-\mathrm{H} 11$ & 120.00 \\
\hline $\mathrm{Ag} 1-\mathrm{Ag} 1-\mathrm{N} 4-\mathrm{C} 9$ & $-71.9(5)$ & $\mathrm{N} 1-\mathrm{C} 1-\mathrm{C} 5-\mathrm{O} 1$ & $-106.9(6)$ \\
\hline $\mathrm{Ag} 1 \mathrm{i}-\mathrm{Ag} 1-\mathrm{N} 4-\mathrm{C} 10$ & $106.2(4)$ & $\mathrm{N} 1-\mathrm{C} 1-\mathrm{C} 5-\mathrm{N} 3$ & $73.3(7)$ \\
\hline $\mathrm{O} 1^{\mathrm{ii}}-\mathrm{Ag} 1-\mathrm{N} 4-\mathrm{C} 9$ & $163.0(5)$ & $\mathrm{C} 1^{\mathrm{v}}-\mathrm{C} 1-\mathrm{C} 5-\mathrm{O} 1$ & $68.8(8)$ \\
\hline $\mathrm{O} 1 \mathrm{ii}-\mathrm{Ag} 1-\mathrm{N} 4-\mathrm{C} 10$ & $-19.0(5)$ & $\mathrm{C} 1 \mathrm{v}-\mathrm{C} 1-\mathrm{C} 5-\mathrm{N} 3$ & $-111.0(7)$ \\
\hline $\mathrm{O} 1^{\mathrm{iii}}-\mathrm{Ag} 1-\mathrm{N} 4-\mathrm{C} 9$ & $53.8(5)$ & $\mathrm{N} 1-\mathrm{C} 1-\mathrm{C} 1^{\mathrm{v}}-\mathrm{N} 1^{\mathrm{v}}$ & $5.5(11)$ \\
\hline $\mathrm{O} 1{ }^{\mathrm{iii}}-\mathrm{Ag} 1-\mathrm{N} 4-\mathrm{C} 10$ & $-128.1(5)$ & $\mathrm{N} 1-\mathrm{C} 1-\mathrm{C} 1^{\mathrm{v}}-\mathrm{C} 5^{\mathrm{v}}$ & $-170.1(6)$ \\
\hline $\mathrm{Ag} 1^{\mathrm{vi}}-\mathrm{O} 1-\mathrm{C} 5-\mathrm{N} 3$ & $-89.1(5)$ & $\mathrm{C} 5-\mathrm{C} 1-\mathrm{C} 1^{\mathrm{v}}-\mathrm{N} 1^{\mathrm{v}}$ & $-170.1(6)$ \\
\hline $\mathrm{Ag} 1^{\mathrm{vi}}-\mathrm{O} 1-\mathrm{C} 5-\mathrm{C} 1$ & $91.1(5)$ & $\mathrm{C} 5-\mathrm{C} 1-\mathrm{C} 1^{\mathrm{v}}-\mathrm{C} 5^{\mathrm{v}}$ & $14.4(10)$ \\
\hline $\mathrm{C} 4-\mathrm{N} 1-\mathrm{C} 1-\mathrm{C} 5$ & $172.9(6)$ & $\mathrm{N} 1-\mathrm{C} 4-\mathrm{C} 4^{\mathrm{v}}-\mathrm{N} 1^{\mathrm{v}}$ & $4.5(11)$ \\
\hline $\mathrm{C} 4-\mathrm{N} 1-\mathrm{C} 1-\mathrm{C} 1^{\mathrm{v}}$ & $-2.9(10)$ & $\mathrm{N} 3-\mathrm{C} 6-\mathrm{C} 7-\mathrm{C} 8$ & $163.0(6)$ \\
\hline $\mathrm{C} 1-\mathrm{N} 1-\mathrm{C} 4-\mathrm{C}^{\mathrm{v}}$ & $-1.9(10)$ & $\mathrm{N} 3-\mathrm{C} 6-\mathrm{C} 7-\mathrm{C} 11$ & $-19.0(9)$ \\
\hline $\mathrm{C} 6-\mathrm{N} 3-\mathrm{C} 5-\mathrm{O} 1$ & $1.7(8)$ & $\mathrm{C} 6-\mathrm{C} 7-\mathrm{C} 8-\mathrm{C} 9$ & $177.0(6)$ \\
\hline $\mathrm{C} 6-\mathrm{N} 3-\mathrm{C} 5-\mathrm{C} 1$ & $-178.5(4)$ & $\mathrm{C} 11-\mathrm{C} 7-\mathrm{C} 8-\mathrm{C} 9$ & $-1.1(9)$ \\
\hline $\mathrm{C} 5-\mathrm{N} 3-\mathrm{C} 6-\mathrm{C} 7$ & $-79.7(7)$ & $\mathrm{C} 6-\mathrm{C} 7-\mathrm{C} 11-\mathrm{C} 10$ & $-177.5(6)$ \\
\hline $\mathrm{Ag} 1-\mathrm{N} 4-\mathrm{C} 9-\mathrm{C} 8$ & $178.2(5)$ & $\mathrm{C} 8-\mathrm{C} 7-\mathrm{C} 11-\mathrm{C} 10$ & $0.5(9)$ \\
\hline $\mathrm{C} 10-\mathrm{N} 4-\mathrm{C} 9-\mathrm{C} 8$ & $0.1(9)$ & $\mathrm{C} 7-\mathrm{C} 8-\mathrm{C} 9-\mathrm{N} 4$ & $0.9(10)$ \\
\hline $\mathrm{Ag} 1-\mathrm{N} 4-\mathrm{C} 10-\mathrm{C} 11$ & $-178.8(5)$ & $\mathrm{N} 4-\mathrm{C} 10-\mathrm{C} 11-\mathrm{C} 7$ & $0.4(9)$ \\
\hline $\mathrm{C} 9-\mathrm{N} 4-\mathrm{C} 10-\mathrm{C} 11$ & $-0.7(9)$ & & \\
\hline
\end{tabular}

Symmetry codes: (i) $x,-y+7 / 4,-z+3 / 4$; (ii) $x-1 / 4, y+1 / 4,-z+1 / 2$; (iii) $-x+2, y+1 / 4, z+1 / 4$; (iv) $-x+7 / 4, y,-z+3 / 4$; (v) $-x+7 / 4,-y+7 / 4, z$; (vi) $-x+2, y-1 / 4$, $z-1 / 4$.

Hydrogen-bond geometry $\left(\AA,{ }^{\circ}\right)$

\begin{tabular}{lllll}
\hline$D-\mathrm{H} \cdots A$ & $D-\mathrm{H}$ & $\mathrm{H} \cdots A$ & $D \cdots A$ & $D-\mathrm{H} \cdots A$ \\
\hline $\mathrm{N} 3-\mathrm{H} 3 N \cdots \mathrm{O} 11^{\text {vii }}$ & 0.88 & 1.86 & $2.744(14)$ & 178 \\
$\mathrm{~N} 3-\mathrm{H} 3 N \cdots \mathrm{O} 13^{\text {vii }}$ & 0.88 & 2.26 & $2.875(13)$ & 127 \\
$\mathrm{C} 4-\mathrm{H} 4 \cdots \mathrm{O} 11^{\text {viii }}$ & 0.95 & 2.45 & $3.378(13)$ & 165 \\
$\mathrm{C} 4-\mathrm{H} 4 \cdots \mathrm{O} 14^{\text {ix }}$ & 0.95 & 2.40 & $3.33(2)$ & 168 \\
$\mathrm{C} 9-\mathrm{H} 9 \cdots \mathrm{O} 13^{\mathrm{x}}$ & 0.95 & 2.50 & $3.224(14)$ & 133 \\
$\mathrm{C} 11-\mathrm{H} 11 \cdots \mathrm{O} 13^{\text {vii }}$ & 0.95 & 2.51 & $3.154(13)$ & 126 \\
\hline
\end{tabular}

Symmetry codes: (vii) $x, y+1, z$; (viii) $-x+2,-y+1,-z$; (ix) $-x+2, y+3 / 4, z-1 / 4$; (x) $x+1 / 4, y+3 / 4,-z+1 / 2$. 